\title{
Stability of a Class of Non-Static Axial Self-Gravitating Systems in $f(R)$ Gravity
}

\author{
M. Sharif ${ }^{1}$ and Z. Yousaf ${ }^{2}$ \\ Department of Mathematics, University of the Punjab, \\ Quaid-e-Azam Campus, Lahore-54590, Pakistan.
}

\begin{abstract}
In this paper, we analyze stability regions of a non-static restricted class of axially symmetric spacetime with anisotropic matter distribution. We consider $f(R)=R+\epsilon R^{2}$ model and assume hydrostatic equilibrium of the axial selfgravitating system at large past time. Considering perturbation from hydrostatic phase, we develop dynamical as well as collapse equations and explore dynamical instabilities at Newtonian and post-Newtonian regimes. It is concluded with the help of stiffness parameter, $\Gamma_{1}$, that radial profile of physical parameters like pressure anisotropy, energy density and higher curvature terms of the $f(R)$ model affect the instability ranges.
\end{abstract}

Subject headings: Axial symmetry; Relativistic fluids; Stability.

\section{Introduction}

Astrophysical latest observations of various experiments like Supernova (Ia) Riess et al. 1998; Perlmutter et al. 1998), weak gravitational lensing of distant galaxies Jain and Taylor (2003), Wilkinson Microwave Anisotropy Probe (Bennett et al. 2003), galactic cluster X-rays emission (Allen et al. 2004) etc indicate the accelerated expansion of the universe. This expansion is assumed to be due to some unusual type of matter referred to dark energy (DE) whose existence can be inferred from its gravitational effects. Many models have been presented to explain the mysterious nature of DE. The cosmic acceleration issue can be better explained using the modified gravity theories such as $f(R), f(R, T), f(G)$ etc. The $f(R)$ gravity theory (Capozziello 2002; Felice and Tsujikawa 2010) is the simplest modification of

\footnotetext{
${ }^{1}$ msharif.math@pu.edu.pk

${ }^{2}$ zeeshan.math@pu.edu.pk
} 
general relativity (GR) obtained by introducing an arbitrary function dependence on the Ricci scalar $R$.

There have been theoretical evidences from several physical processes that show the importance of local anisotropies in pressure (Herrera and Santos 2010; Mak and Harko 2003). Herrera and Santos (1995) analyzed the role of slow rotation in the astronomical anisotropic spherical stars. Weber (1999) studied that strong magnetic fields within the compact spheres cause to generate pressure anisotropy. Chakraborty et al. (2005) examined the effects of tangential as well as radial components of pressure in the collapse of relativistic objects within quasi-spherical model.

The analysis on the final collapse phase is of great interest for many astrophysicists. Oppenheimer and Snyder (1939) explored collapsing process of a homogenous dust cloud which gives rise to black hole $(\mathrm{BH})$. Misner and Sharp (1964) analyzed perfect matter distribution collapse and concluded that its final phase will be a BH. Garattini (2009) examined the naked singularity formation with mass parameter in $f(R)$ background. Ziaie et al. (2011) investigated the evolution of collapsing mechanisms in stellar bodies with $\epsilon R^{m}$ gravity. Recently, Sharif and his collaborators (Sharif and Bhatti 2012a, b; ; Sharif and Yousaf 2012a, b) have examined the dynamics of collapsing compact bodies and provided several interesting results through a relationship between inhomogeneous energy density and Weyl tensor.

The study of gravitational collapse has also been investigated in modified gravity theories. Cognola et al. (2005) worked on BH solution of the spherical collapsing body in $f(R)$ gravity with a positive constant Ricci curvature condition. Cruz-Dombriz et al. (2009) explored the collapsing sphere and presented BH solutions with negative constant Ricci scalar scenario. Bergliaffa and Nunes (2011) studied spherical BH models in the context of nonconstant Ricci scalar condition. Sharif and Yousaf (2013) investigated the collapsing spherical astronomical fluid distribution in $f(R)$ gravity and concluded that the $f(R)$ gravity terms delay the black hole formation.

The dynamical instability of astronomical spherical objects like supermassive stars against radial perturbations and their final phase has been a subject of keen interest. Chandrasekhar (1964) presented a formalism to discuss the dynamical instability of the isotropic ideal collapsing sphere. Herrera et al. (1989) explored the stability of the spherical dissipative collapsing body and found that heat dissipation makes the system stable. Many authors (Chan et al. 1993, 1994; Chan 2000) examined the role of anisotropic pressure, viscosity and radiation density on the instability of spherical collapse at both Newtonian (N) as well as post-Newtonian (pN) approximations. Matarrese and Terranova (1996) described the pN cosmological dynamics of the irrotational dust cloud. Capozziello et al. (2011) explored modified Lané-Emden equation through $\mathrm{N}$ limit of metric $f(R)$ gravity and analyzed 
hydrostatic phases of stellar structures.

Capozziello et al. (2012) investigated Jeans instability for self-gravitating stellar systems through weak field approximation $f(R)$ gravity coupled with ideal matter distribution. Sharif and his collaborators (Sharif and Bhatti 2013a, 2014, 2013b; Sharif and Yousaf 2013a) discussed the instability ranges of the collapsing stars and found that kinematical quantities like the higher curvature terms of $f(R)$ gravity, shearfree condition etc cause the system less unstable thereby slowing down the collapse rate. Farinelli et al. (2014) numerically solved modified Lané-Emden equation in order to discuss the stability of some unexplored exotic stellar structures in $f(R)$ background.

To explore the mysterious phenomenon of dark universe, variety of $f(R)$ models are extensively studied. Copeland et al. (2006) discussed various formalisms to discuss expansion of accelerating universe. Nojiri and Odintsov (2007) suggested several generic functions of $f(R, G)$ and $f(R)$ gravity theories to analyzed dark aspects of Universe during late-time era. Amendola et al. (2007) presented some viable $f(R)$ gravity models in both Einstein and Jordan frames. Cognola et al. (2008) proposed exponential-type model which provides the accelerating cosmic solutions lacking a future singularity.

Nojiri and Odintsov (2011) introduced some well-consistent $f(R)$ models describing both inflation as well as late-time acceleration of the universe. The more interesting $f(R)$ models along with their viable conditions have been proposed by Cruz-Dombriz, A.D.L. and Sáez-Gómez (2012). Cembranos et al. (2012) claimed that the study of gravitational collapse in the presence of dark source $f(R)$ terms gives an interesting results about the cosmic acceleration. Therefore, it would be very worthwile to discuss the dynamical instability regions of collapsing systems with $f(R)$ models.

Capozziello and Laurentis (2011) presented several solutions of axially symmetric body with the help of spherical configuration of matter in $f(R)$ gravity. Herrera et al. (2013) found axially symmetric analytic solutions and concluded that under spherical limit, their solution represent the Schwarzschild metric. Nojiri and Odintsov (2013) proved that inflationary and/or dark energy higher curvature $f(R)$ terms support the anti-evaporation of Schwarzschild-de Sitter black hole on classical level. Sebastiani et al. (2013) used cosmological patch technique to discuss Nariai black holes future evolution with power-law $f(R)$ theory and concluded that instabilities of such stellar structure depends upon the specific choice of model.

In this paper, we perform stellar stability analysis for a non-static axially symmetric restricted spacetime with anisotropic matter configuration through the perturbation technique in $f(R)$ gravity. The outline of the paper as follows. In the next section, we develop the 
field equations as well as dynamical equations for anisotropic fluid distribution with viable $f(R)$ model. In section $\mathbf{3}$, we formulate collapse equation using perturbation scheme while section 4 establishes the instability ranges in the $\mathrm{N}$ and $\mathrm{pN}$ regimes. In the last section, we conclude our results.

\section{Anisotropic Source and Field Equations}

The Einstein-Hilbert action in $f(R)$ gravity can be written as

$$
S_{f(R)}=\frac{1}{2 \kappa} \int d^{4} x \sqrt{-g} f(R)+S_{M},
$$

where $f(R)$ is a non-linear real function of the curvature $R, \kappa$ is the coupling constant and $S_{M}$ is the matter action. The corresponding field equations in metric formalism are

$$
f_{R} R_{\alpha \beta}-\frac{1}{2} f g_{\alpha \beta}-\nabla_{\alpha} \nabla_{\beta} f_{R}+g_{\alpha \beta} \square f_{R}=\kappa T_{\alpha \beta},
$$

where $\nabla_{\alpha}$ indicates covariant derivative and $\square=\nabla^{\alpha} \nabla_{\alpha}$. We can develop a formulation in the form of Einstein field equations as

$$
G_{\alpha \beta}=\frac{\kappa}{f_{R}}\left(\stackrel{(D)}{\alpha \beta}_{\alpha \beta}+T_{\alpha \beta}\right)
$$

where

$$
\stackrel{(D)}{T_{\alpha \beta}}=\frac{1}{\kappa}\left\{\frac{f-R f_{R}}{2} g_{\alpha \beta}+\nabla_{\alpha} \nabla_{\beta} f_{R}-\square f_{R} g_{\alpha \beta}\right\},
$$

is the effective stress energy-momentum tensor. The trace of Eq.(1) yields

$$
R f_{R}+3 \square f_{R}-2 f=\kappa T,
$$

which under constant curvature condition gives

$$
R f_{R}-2 f=0
$$

This represents de-Sitter point, a vacuum solution, i.e., $T=0$.

The most general axial symmetric metric in spherical coordinates can be reduced to the form (Roy and Tripathi 1971)

$$
d s^{2}=-A^{2}(t, r, \theta) d t^{2}+B^{2}(t, r, \theta)\left(d r^{2}+r^{2} d \theta^{2}\right)+C^{2}(t, r, \theta) d \phi^{2} .
$$

This is the restricted non-static axial symmetry since we have ignored the terms describing reflection as well as those characterizing the rotations about the symmetry axis, i.e., $d t d \phi$. The 
consideration of "rotation" and "reflection" along with four independent metric coefficients in $f(R)$ gravity could not be handled analytically. We have excluded such terms in our metric just for the sake of simplicity. It is worthy to mention that recently, Herrera et al. (2014) have examined dissipative gravitational collapse of non- static axially symmetric spacetime in GR. They performed analysis by restricting the axial symmetry to reflection and neglecting the meridional motions. We consider that the collapsing axial symmetric body is filled with anisotropic matter whose energy-momentum tensor is (Herrera et al. 2013)

$$
\begin{aligned}
T_{\alpha \beta} & =(\mu+P) V_{\alpha} V_{\beta}-\left(K_{\alpha} K_{\beta}-\frac{1}{3} h_{\alpha \beta}\right)\left(P_{z z}-P_{x x}\right)+P g_{\alpha \beta} \\
& -\left(P_{z z}-P_{y y}\right)\left(L_{\alpha} L_{\beta}-\frac{1}{3} h_{\alpha \beta}\right)+2 K_{(\alpha} L_{\beta)} P_{x y}
\end{aligned}
$$

where

$$
h_{\alpha \beta}=g_{\alpha \beta}+V_{\alpha} V_{\beta}, \quad P=\frac{1}{3}\left(P_{y y}+P_{x x}+P_{z z}\right),
$$

$\mu$ is the energy density and $P_{x x}, P_{y y}, P_{z z}$ are the different pressures with $P_{y y} \neq P_{x x} \neq P_{z z}$ and $P_{x y}=P_{y x}$. Also, $V_{\alpha}, K_{\alpha}$ and $L_{\alpha}$ are the four velocity, unit four-vectors, respectively and $\alpha, \beta$ are the Lorentz indices. In comoving coordinate system, we have

$$
V_{\beta}=-A \delta_{\beta}^{0}, \quad L_{\beta}=r B \delta_{\beta}^{2}, \quad K_{\beta}=B \delta_{\beta}^{1} .
$$

The quantities controlling the kinematics of the matter distribution are the shear tensor $\sigma_{\alpha \beta}$, the four acceleration $a_{\alpha}$ and the expansion scalar $\Theta$. The non-zero values of these variables are given as

$$
\begin{aligned}
a_{1} & =\frac{A^{\prime}}{A}, \quad a_{2}=\frac{A^{\theta}}{A}, \quad \Theta=\frac{1}{A}\left(\frac{\dot{C}}{C}+\frac{2 \dot{B}}{B}\right), \quad \sigma_{11}=\frac{\sigma}{3} B^{2}, \\
\sigma_{22} & =\frac{\sigma}{3} r^{2} B^{2}, \quad \sigma_{33}=-\frac{2 \sigma}{3} C^{2}, \quad \sigma=-\frac{1}{A}\left(\frac{\dot{C}}{C}-\frac{\dot{B}}{B}\right),
\end{aligned}
$$

where dot, prime and superscript $\theta$ represent derivatives with respect to time, radius and $\theta$, respectively. The corresponding Ricci scalar becomes

$$
\begin{aligned}
R & =2\left[\frac { 1 } { B ^ { 2 } } \left\{\frac{A^{\prime \prime}}{A}+\frac{A^{\prime} C^{\prime}}{A C}+\frac{B^{\prime \prime}}{B}+\frac{1}{r}\left(\frac{C^{\prime}}{C}+\frac{B^{\prime}}{B}-\frac{A^{\prime}}{A}\right)-\frac{B^{\prime 2}}{B^{2}}+\frac{C^{\prime \prime}}{C}\right.\right. \\
& \left.+\frac{1}{r^{2}}\left(\frac{B^{\theta \theta}}{B}-\frac{B^{\theta 2}}{B^{2}}+\frac{A^{\theta \theta}}{A}+\frac{C^{\theta \theta}}{C}+\frac{A^{\theta} C^{\theta}}{A C}\right)\right\} \\
& \left.+\frac{1}{A^{2}}\left\{\frac{\dot{A}}{A}\left(\frac{2 \dot{B}}{B}+\frac{\dot{C}}{C}\right)-\frac{\dot{B}}{B}\left(\frac{\dot{C}}{C}+\frac{\dot{B}}{B}\right)-\frac{2 \ddot{B}}{B}-\frac{\ddot{C}}{C}\right\}\right]
\end{aligned}
$$


The corresponding $f(R)$ field equations take the form

$$
\begin{aligned}
& \frac{\dot{B}}{B A^{2}}\left(\frac{2 \dot{C}}{C}+\frac{\dot{B}}{B}\right)-\frac{1}{B^{2}}\left[\frac{1}{r}\left(\frac{C^{\prime}}{C}+\frac{B^{\prime}}{B}\right)-\left(\frac{B^{\prime}}{B}\right)^{2}+\frac{B^{\prime \prime}}{B}+\frac{C^{\prime \prime}}{C}+\frac{1}{r^{2}}\right. \\
& \left.\times\left\{\frac{C^{\theta \theta}}{C}-\left(\frac{B^{\theta}}{B}\right)^{2}+\frac{B^{\theta \theta}}{B}\right\}\right]=\frac{\kappa}{f_{R}}\left[\mu+\frac{1}{\kappa}\left\{\frac{f-R f_{R}}{2}+\frac{f_{R}^{\prime \prime}}{B^{2}}-\frac{\dot{f_{R}}}{A^{2}}\left(\frac{2 \dot{B}}{B}\right.\right.\right. \\
& \left.\left.\left.+\frac{\dot{C}}{C}\right)+\frac{f_{R}^{\prime}}{B^{2}}\left(\frac{C^{\prime}}{C}-\frac{2 B^{\prime}}{B}+\frac{1}{r}\right)+\frac{f_{R}^{\theta}}{B^{2} r^{2}}\left(\frac{C^{\theta}}{C}-\frac{2 B^{\theta}}{B}\right)+\frac{f_{R}^{\theta \theta}}{B^{2} r^{2}}\right\}\right], \\
& \frac{A^{\prime}}{A}\left(\frac{\dot{B}}{B}+\frac{\dot{C}}{C}\right)+\frac{\dot{B}}{B}\left(\frac{B^{\prime}}{B}+\frac{C^{\prime}}{C}\right)-\frac{\dot{B}^{\prime}}{B}-\frac{\dot{C}^{\prime}}{C}=\frac{\dot{f}_{R}^{\prime}}{f_{R}}-\frac{A^{\prime} \dot{f}_{R}}{A f_{R}}-\frac{\dot{B} f_{R}^{\prime}}{B f_{R}}, \\
& \frac{A^{\theta}}{A}\left(\frac{\dot{B}}{A}+\frac{\dot{C}}{C}\right)+\frac{\dot{B}}{B}\left(\frac{B^{\theta}}{B}+\frac{C^{\theta}}{C}\right)-\frac{\dot{B}^{\theta}}{B}-\frac{\dot{C}^{\theta}}{C}=\frac{\dot{f}_{R}{ }^{\theta}}{f_{R}}-\frac{A^{\theta} \dot{f}_{R}}{A f_{R}}-\frac{\dot{B} f_{R}^{\theta}}{B f_{R}}, \\
& \frac{1}{A^{2}}\left[\frac{\dot{B} \dot{A}}{B A}-\frac{\ddot{C}}{C}-\frac{\ddot{B}}{B}+\frac{\dot{C}}{C}\left(\frac{\dot{A}}{A}-\frac{\dot{B}}{C}\right)\right]+\frac{1}{B^{2}}\left[\frac{A^{\prime} B^{\prime}}{A B}+\frac{C^{\prime}}{C}\left(\frac{A^{\prime}}{A}+\frac{B^{\prime}}{B}\right)\right. \\
& \left.+\frac{1}{r}\left(\frac{C^{\prime}}{C}+\frac{A^{\prime}}{A}\right)+\frac{1}{r^{2}}\left\{\frac{A^{\theta \theta}}{A}+\frac{C^{\theta}}{C}\left(\frac{A^{\theta}}{A}-\frac{B^{\theta}}{B}\right)+\frac{C^{\theta \theta}}{C}-\frac{A^{\theta} B^{\theta}}{A B}\right\}\right] \\
& =\frac{\kappa}{f_{R}}\left[P_{x x}-\frac{1}{\kappa}\left\{\frac{f-R f_{R}}{2}-\frac{\ddot{f}_{R}}{A^{2}}+\frac{f_{R}^{\theta \theta}}{B^{2} r^{2}}+\frac{\dot{f_{R}}}{A^{2}}\left(\frac{\dot{A}}{A}-\frac{\dot{B}}{B}+\frac{\dot{C}}{C}\right)+\frac{f_{R}^{\prime}}{B^{2}}\right.\right. \\
& \left.\left.\times\left(\frac{A^{\prime}}{A}-\frac{B^{\prime}}{B}-\frac{1}{r}+\frac{C^{\prime}}{C}\right)+\frac{f_{R}^{\theta}}{B^{2} r^{2}}\left(\frac{A^{\theta}}{A}-\frac{3 B^{\theta}}{B}+\frac{C^{\theta}}{C}\right)\right\}\right], \\
& \frac{1}{A^{2}}\left[\frac{\dot{B} \dot{A}}{B A}-\frac{\ddot{C}}{C}-\frac{\ddot{B}}{B}+\frac{\dot{C}}{C}\left(\frac{\dot{A}}{A}-\frac{\dot{B}}{B}\right)\right]+\frac{1}{B^{2}}\left[\frac{C^{\prime \prime}}{C}+\frac{A^{\prime \prime}}{A}-\frac{A^{\prime} B^{\prime}}{A B}+\frac{C^{\prime}}{C}\right. \\
& \left.\times\left(\frac{A^{\prime}}{A}-\frac{B^{\prime}}{B}\right)+\frac{1}{r^{2}}\left\{\frac{B^{\theta} A^{\theta}}{B A}+\frac{C^{\theta}}{C}\left(\frac{A^{\theta}}{A}+\frac{B^{\theta}}{B}\right)\right\}\right]=\frac{\kappa}{f_{R}}\left[P_{y y}-\frac{1}{\kappa}\right. \\
& \times\left\{\frac{f-R f_{R}}{2}-\frac{\ddot{f_{R}}}{A^{2}}+\frac{f_{R}^{\prime \prime}}{B^{2}}+\frac{\dot{f_{R}}}{A^{2}}\left(\frac{\dot{A}}{A}-\frac{\dot{B}}{B}-\frac{\dot{C}}{C}\right)+\frac{f_{R}^{\prime}}{B^{2}}\left(\frac{A^{\prime}}{A}-\frac{B^{\prime}}{B}+\frac{C^{\prime}}{C}\right)\right. \\
& \left.\left.+\frac{f_{R}^{\theta}}{B^{2} r^{2}}\left(\frac{A^{\theta}}{A}-\frac{B^{\theta}}{B}+\frac{C^{\theta}}{C}\right)\right\}\right] \\
& \frac{1}{A^{2}}\left[\frac{\dot{B}}{B}\left(\frac{\dot{A}}{A}+\frac{\dot{B}}{B}\right)+\frac{2 \ddot{B}}{B}\right]+\frac{1}{B^{2}}\left[\frac{B^{\prime \prime}}{B}+\frac{A^{\prime \prime}}{A}-\left(\frac{B^{\prime}}{B}\right)^{2}+\frac{1}{r}\left(\frac{B^{\prime}}{B}+\frac{A^{\prime}}{A}\right)\right. \\
& \left.+\frac{1}{r^{2}}\left\{\frac{B^{\theta \theta}}{B}+\frac{A^{\theta \theta}}{A}-\frac{B^{\theta 2}}{B^{2}}\right\}\right]=\frac{\kappa}{f_{R}}\left[P_{z z}-\frac{1}{\kappa}\left\{\frac{f-R f_{R}}{2}-\frac{\ddot{f_{R}}}{A^{2}}+\frac{f_{R}^{\prime \prime}}{B^{2}}+\frac{f_{R}^{\theta \theta}}{B^{2} r^{2}}\right.\right.
\end{aligned}
$$




$$
\begin{aligned}
& \left.\left.+\frac{\dot{f_{R}}}{A^{2}}\left(\frac{\dot{A}}{A}-\frac{2 \dot{B}}{B}\right)+\frac{f_{R}^{\prime}}{B^{2}}\left(\frac{A^{\prime}}{A}-\frac{2 B^{\prime}}{B}-\frac{1}{r}\right)+\frac{f_{R}^{\theta \theta}}{B^{2} r^{2}}\left(\frac{A^{\theta}}{A}-\frac{2 B^{\theta}}{B}\right)\right\}\right] \\
& \frac{1}{B^{2}}\left[\frac{1}{r}\left\{-\frac{C^{\prime}}{C}-\frac{A^{\prime} \theta}{A}+\frac{B^{\prime}}{B}\left(\frac{A^{\theta}}{A}+\frac{C^{\theta}}{C}\right)+\frac{B^{\theta}}{B}\left(\frac{C^{\prime}}{C}+\frac{A^{\prime}}{A}\right)\right\}\right. \\
& \left.+\left(\frac{C^{\theta}}{C}+\frac{A^{\theta}}{A}\right) \frac{1}{r^{2}}\right]=\frac{\kappa}{f_{R}}\left[P_{x y}+\frac{1}{\kappa}\left(f_{R}^{\prime \theta}-\frac{B^{\theta} f_{R}^{\prime}}{B}-\frac{f_{R}^{\theta}}{r}-\frac{B^{\prime} f_{R}^{\theta}}{B}\right)\right] .
\end{aligned}
$$

The dynamical equations describe how parameters of the self-gravitating collapsing objects evolve with time and radius. The corresponding dynamical equations can be found through

$$
\left(T^{\alpha \beta}+T^{(D)}\right)_{; \beta} V_{\alpha}=0, \quad\left(T^{\alpha \beta}+T^{\alpha \beta}\right)_{; \beta} L_{\alpha}=0, \quad\left(T^{\alpha \beta}+T^{(D)}\right)_{; \beta} K_{\alpha}=0
$$

which lead to

$$
\begin{aligned}
& \frac{\dot{\mu}}{A}+\left(\mu+P_{z z}\right) \frac{\dot{C}}{A C}+\frac{\dot{B}}{A B}\left(2 \mu+P_{y y}+P_{x x}\right)+\frac{D_{0}(t, r, \theta)}{\kappa A}=0, \\
& \frac{P_{x x}^{\prime}}{B}+\frac{A^{\prime}}{A B}\left(P_{x x}+\mu\right)-\frac{B^{\prime}}{B^{2}}\left(P_{y y}-P_{x x}\right)-\frac{C^{\prime}}{B C}\left(P_{z z}-P_{x x}\right)+\frac{1}{r B} \\
& \times\left\{P_{x y}\left(\frac{C^{\theta}}{C}+\frac{2 B^{\theta}}{B}+\frac{A^{\theta}}{A}\right)+P_{x y}^{\theta}-P_{y y}+P_{x x}\right\}+\frac{D_{1}(t, r, \theta)}{\kappa B}=0, \\
& \frac{r P_{y y}^{\theta}}{B}+\frac{r A^{\theta}}{A B}\left(P_{y y}+\mu\right)-\frac{r B^{\theta}}{B^{2}}\left(P_{x x}-P_{y y}\right)-\frac{r C^{\theta}}{B C}\left(P_{z z}-P_{y y}\right)+\frac{2 r P_{x y}}{B} \\
& +\left[P_{x y}^{\prime}+P_{x y}\left(\frac{C^{\prime}}{C}+\frac{2 B^{\prime}}{B}+\frac{A^{\prime}}{A}\right)\right] \frac{r^{2}}{B}+\frac{r D_{2}(t, r, \theta)}{\kappa B}=0,
\end{aligned}
$$

where $D_{0}, D_{1}$ and $D_{2}$ are given in Appendix A. Many inflation models in the early universe are established on scalar fields coming from supergravity and superstring theories. The first model of inflation was suggested by Starobinsky which corresponds to the conformal anomaly in quantum gravity (Capozziello and Laurentis 2011) given by

$$
f(R)=R+\epsilon R^{2}
$$

This model was suggested both as a model for dark matter (Cembranos et al. 2012) as well as an inflationary candidate (Starobinsky 1980) which can lead to the accelerated universe expansion due to $R^{2}$ term. It is mentioned here that $\epsilon=\frac{1}{6 M^{2}}$ which is proposed for dark matter model. It is worth mentioning here that the value of $M$ is figured out as $2.7 \times 10^{-12} \mathrm{GeV}$ with $\epsilon \leq 2.3 \times 10^{22} \mathrm{Ge} / V^{2}$ (Sharif and Yousaf 2014a). General relativity is recovered for $\epsilon=0$ that corresponds to classically stable BH (Sotirou and Faraoni 2010). In $f(R)$ gravity, 
all these characteristics are also observed thus the stability condition for this theory takes the form $[\epsilon(1+2 \epsilon R)]^{-1} \geq 0$. Recently, slow-roll inflation has been discussed through $f(R)$ polynomial models (Huang 2014; Sharif and Yousaf 2014b).

\section{Perturbation Scheme}

Here, we use the perturbation technique (Herrera et al. 1989) to perturb the dynamical equations and Ricci scalar upto first order in $\alpha$, where $0<\alpha \ll 1$. The system is assumed to be entirely in the state of hydrostatic equilibrium, but upon evolution, time dependence factor $T(t)$ appears in all the functions that are controlling the kinematics of the system. We further consider that all the metric coefficients possess the same time dependence that imparts similar time dependence on the Ricci scalar. Notice that $T(t)$ is not a trace of the energy-momentum tensor instead an arbitrary function of time. The fluid and metric variables are perturbed as

$$
\begin{aligned}
A(t, r, \theta) & =A_{0}(r, \theta)+\alpha T(t) a(r, \theta), \\
B(t, r, \theta) & =B_{0}(r, \theta)+\alpha T(t) b(r, \theta), \\
C(t, r, \theta) & =C_{0}(r, \theta)+\alpha T(t) c(r, \theta), \\
\mu(t, r, \theta) & =\mu_{0}(r, \theta)+\alpha \bar{\mu}(t, r, \theta), \\
P_{x y}(t, r, \theta) & =P_{x y 0}(r, \theta)+\alpha \bar{P}_{x y}(t, r, \theta), \\
P_{x x}(t, r, \theta) & =P_{x x 0}(r, \theta)+\alpha \bar{P}_{x x}(t, r, \theta), \\
P_{y y}(t, r, \theta) & =P_{y y 0}(r, \theta)+\alpha \bar{P}_{y y}(t, r, \theta), \\
P_{z z}(t, r, \theta) & =P_{z z 0}(r, \theta)+\alpha \bar{P}_{z z}(t, r, \theta), \\
R(t, r, \theta) & =R_{0}(r, \theta)+\alpha T(t) e(r, \theta), \\
f(t, r, \theta) & =R_{0}\left(1+\epsilon R_{0}\right)+\alpha T(t) e(r, \theta)\left(1+2 \epsilon R_{0}\right), \\
f_{R}(t, r, \theta) & =\left(1+2 \epsilon R_{0}\right)+2 \alpha \epsilon T(t) e(r, \theta),
\end{aligned}
$$

where $R_{0}$ is the static part of Eq.(6) and is computed as

$$
\begin{aligned}
& R_{0}=\frac{2}{B_{0}^{2}}\left\{\frac{C_{0}^{\prime \prime}}{C_{0}}+\frac{B_{0}^{\prime \prime}}{B_{0}}+\frac{A_{0}^{\prime} C_{0}^{\prime}}{A_{0} C_{0}}+\frac{A_{0}^{\prime \prime}}{A_{0}}+\frac{1}{r}\left(-\frac{A_{0}^{\prime}}{A_{0}}+\frac{C_{0}^{\prime}}{C_{0}}+\frac{B_{0}^{\prime}}{B_{0}}\right)-\frac{B_{0}^{\prime 2}}{B_{0}^{2}}\right. \\
& \left.+\frac{1}{r^{2}}\left(\frac{C_{0}^{\theta \theta}}{C_{0}}+\frac{B_{0}^{\theta \theta}}{B_{0}}+\frac{A_{0}^{\theta \theta}}{A_{0}}+\frac{C_{0}^{\theta} A_{0}^{\theta}}{A_{0} C_{0}}-\frac{B_{0}^{\theta 2}}{B_{0}^{2}}\right)\right\} .
\end{aligned}
$$

The static part of the field equations (77)-(13) are obtained by using Eqs.(18)-(28) as 
follows

$$
\begin{aligned}
& -\frac{1}{B_{0}^{2}}\left[\frac{B_{0}^{\prime \prime}}{B_{0}}-\left(\frac{B_{0}^{\prime}}{B_{0}}\right)^{2}+\frac{1}{r}\left(\frac{B_{0}^{\prime}}{B_{0}}+\frac{C_{0}^{\prime}}{C_{0}}\right)+\frac{C_{0}^{\prime \prime}}{C_{0}} \frac{1}{r^{2}}\left\{\frac{B_{0}^{\theta \theta}}{B_{0}}-\left(\frac{B_{0}^{\theta}}{B_{0}}\right)^{2}\right.\right. \\
& \left.\left.+\frac{C_{0}^{\theta \theta}}{C_{0}}\right\}\right]=\frac{\kappa}{1+2 \epsilon R_{0}}\left[\mu_{0}+\frac{2 \epsilon}{\kappa}\left\{-\frac{R_{0}^{2}}{4}+\frac{R_{0}^{\prime \prime}}{B_{0}^{2}}+\frac{R_{0}^{\prime}}{B_{0}^{2}}\left(\frac{C_{0}^{\prime}}{C_{0}}-\frac{2 B_{0}^{\prime}}{B_{0}}\right.\right.\right. \\
& \left.\left.\left.+\frac{1}{r}\right)+\frac{R_{0}^{\theta}}{B_{0}^{2} r^{2}}\left(\frac{C_{0}^{\theta}}{C_{0}}-\frac{2 B_{0}^{\theta}}{B_{0}}\right)+\frac{R_{0}^{\theta \theta}}{B_{0}^{2} r^{2}}\right\}\right] \text {, } \\
& \frac{1}{B_{0}^{2}}\left[\frac{B_{0}^{\prime} A_{0}^{\prime}}{A_{0} B_{0}}+\frac{C_{0}^{\prime}}{C_{0}}\left(\frac{A_{0}^{\prime}}{A_{0}}+\frac{B_{0}^{\prime}}{B_{0}}\right)+\frac{1}{r}\left(\frac{C_{0}^{\prime}}{C_{0}}+\frac{A_{0}^{\prime}}{A_{0}}\right)+\frac{1}{r^{2}}\left\{\frac{C_{0}^{\theta \theta}}{C_{0}}+\frac{A_{0}^{\theta \theta}}{A_{0}}+\frac{C_{0}^{\theta}}{C_{0}}\right.\right. \\
& \left.\left.\times\left(\frac{A_{0}^{\theta}}{A_{0}}-\frac{B_{0}^{\theta}}{B_{0}}\right)-\frac{A_{0}^{\theta} B_{0}^{\theta}}{A_{0} B_{0}}\right\}\right]=\frac{\kappa}{1+2 \epsilon R_{0}}\left[P_{x x 0}-\frac{2 \epsilon}{\kappa}\left\{-\frac{R_{0}^{2}}{4}+\frac{R_{0}^{\theta \theta}}{B_{0}^{2} r^{2}}\right.\right. \\
& \left.\left.+\frac{R_{0}^{\prime}}{B_{0}^{2}}\left(\frac{A_{0}^{\prime}}{A_{0}}-\frac{B_{0}^{\prime}}{B_{0}}-\frac{1}{r}+\frac{C_{0}^{\prime}}{C_{0}}\right)+\frac{R_{0}^{\theta}}{B_{0}^{2} r^{2}}\left(\frac{A_{0}^{\theta}}{A_{0}}-\frac{3 B_{0}^{\theta}}{B_{0}}+\frac{C_{0}^{\theta}}{C_{0}}\right)\right\}\right], \\
& \frac{1}{B_{0}^{2}}\left[\frac{C_{0}^{\prime \prime}}{C_{0}}+\frac{A_{0}^{\prime \prime}}{A_{0}}+\frac{C_{0}^{\prime}}{C_{0}}\left(\frac{A_{0}^{\prime}}{A_{0}}-\frac{B_{0}^{\prime}}{B_{0}}\right)-\frac{A_{0}^{\prime} B_{0}^{\prime}}{A_{0} B_{0}}+\frac{1}{r^{2}}\left\{\frac{B_{0}^{\theta} A_{0}^{\theta}}{A_{0} B_{0}}+\frac{C_{0}^{\theta}}{C_{0}}\left(\frac{A_{0}^{\theta}}{A_{0}}\right.\right.\right. \\
& \left.\left.\left.+\frac{B_{0}^{\theta}}{B_{0}}\right)\right\}\right]=\frac{\kappa}{1+2 \epsilon R_{0}}\left[P_{y y 0}+\frac{2 \epsilon}{\kappa}\left\{\frac{R_{0}^{2}}{4}-\frac{R_{0}^{\prime \prime}}{B_{0}^{2}}-\frac{R_{0}^{\prime}}{B_{0}^{2}}\left(\frac{A_{0}^{\prime}}{A_{0}}-\frac{B_{0}^{\prime}}{B_{0}}+\frac{C_{0}^{\prime}}{C_{0}}\right)\right.\right. \\
& \left.\left.-\frac{R_{0}^{\theta}}{B_{0}^{2} r^{2}}\left(\frac{A_{0}^{\theta}}{A_{0}}-\frac{B_{0}^{\theta}}{B_{0}}+\frac{C_{0}^{\theta}}{C_{0}}\right)\right\}\right] \text {, } \\
& \frac{1}{B_{0}^{2}}\left[\frac{B_{0}^{\prime \prime}}{B_{0}}+\frac{A_{0}^{\prime \prime}}{A_{0}}-\left(\frac{B_{0}^{\prime}}{B_{0}}\right)^{2}+\frac{1}{r}\left(\frac{B_{0}^{\prime}}{B_{0}}+\frac{A_{0}^{\prime}}{A_{0}}\right)+\frac{1}{r^{2}}\left\{\frac{B_{0}^{\theta \theta}}{B_{0}}+\frac{A_{0}^{\theta \theta}}{A_{0}}-\frac{B_{0}^{\theta 2}}{B_{0}^{2}}\right\}\right] \\
& =\frac{\kappa}{1+2 \epsilon R_{0}}\left[P_{z z 0}-\frac{2 \epsilon}{\kappa}\left\{-\frac{R_{0}^{2}}{4}+\frac{R_{0}^{\theta \theta}}{B_{0}^{2}}+\frac{R_{0}^{\prime \prime}}{B_{0}^{2}}+\frac{R_{0}^{\prime}}{B_{0}^{2}}\left(\frac{A_{0}^{\prime}}{A_{0}}-\frac{2 B_{0}^{\prime}}{B_{0}}-\frac{1}{r}\right)\right.\right. \\
& \left.\left.+\frac{R_{0}^{\theta}}{B_{0}^{2} r^{2}}\left(\frac{A_{0}^{\theta}}{A_{0}}-\frac{2 B_{0}^{\theta}}{B_{0}}\right)\right\}\right] \text {, } \\
& \frac{1}{B_{0}^{2}}\left[\frac{1}{r}\left\{-\frac{A_{0}^{\prime} \theta}{A_{0}}+\frac{B_{0}^{\prime} C_{0}^{\theta}}{B_{0} C_{0}}-\frac{C_{0}^{\prime} \theta}{C_{0}}+\frac{B_{0}^{\theta}}{B_{0}}\left(\frac{A_{0}^{\prime}}{A_{0}}+\frac{C_{0}^{\prime}}{C_{0}}\right)+\frac{A_{0}^{\theta} B_{0}^{\prime}}{A_{0} B_{0}}\right\}+\frac{1}{r^{2}}\left(\frac{A_{0}^{\theta}}{A_{0}}\right.\right. \\
& \left.\left.+\frac{C_{0}^{\theta}}{C_{0}}\right)\right]=\frac{\kappa}{1+2 \epsilon R_{0}}\left[P_{x y 0}+\frac{2 \epsilon}{\kappa}\left\{R_{0}^{\prime \theta}-\frac{B_{0}^{\theta}}{B_{0}} R_{0}^{\prime}-\frac{R_{0}^{\theta}}{r}-\frac{B_{0}^{\prime}}{B_{0}} R_{0}^{\theta}\right\}\right] \text {. }
\end{aligned}
$$

The dynamical equations (14)-(16) under static background leads to

$$
\begin{aligned}
& P_{x x 0}^{\prime}+\left(P_{x x 0}+\mu_{0}\right) \frac{A_{0}^{\prime}}{A_{0}}-\left(P_{y y 0}-P_{x x 0}\right) \frac{B_{0}^{\prime}}{B_{0}}-\left(P_{z z 0}-P_{x x 0}\right) \frac{C_{0}^{\prime}}{C_{0}}+\frac{P_{x y 0}}{r} \\
& \times\left(\frac{C_{0}^{\theta}}{C_{0}}+\frac{2 B_{0}^{\theta}}{B_{0}}+\frac{A_{0}^{\theta}}{A_{0}}\right)+\frac{1}{r}\left(P_{x y 0}^{\theta}-P_{y y 0}+P_{x x 0}\right)+\frac{D_{1 S}(r, \theta)}{\kappa}=0, \\
& P_{y y 0}^{\theta}+\left(P_{y y 0}+\mu_{0}\right) \frac{A_{0}^{\theta}}{A_{0}}-\left(P_{x x 0}-P_{y y 0}\right) \frac{B_{0}^{\theta}}{B_{0}}-\left(P_{z z 0}-P_{y y 0}\right) \frac{C_{0}^{\theta}}{C_{0}}
\end{aligned}
$$




$$
+2 P_{x y 0}+r\left\{P_{x y 0}\left(\frac{C_{0}^{\prime}}{C_{0}}+\frac{2 B_{0}^{\prime}}{B_{0}}+\frac{A_{0}^{\prime}}{A_{0}}\right)+P_{x y 0}^{\prime}\right\}+\frac{D_{2 S}(r, \theta)}{\kappa}=0,
$$

where $D_{1 S}$ and $D_{2 S}$ are static parts of the above equations given in Appendix $\mathbf{A}$.

The perturbed form of Eqs.(14)-(16) will be

$$
\begin{aligned}
& \dot{\bar{\mu}}+\left\{\frac{c}{C_{0}}\left(P_{z z 0}+\mu_{0}\right)+\left(2 \mu_{0}+P_{x x 0}+P_{y y 0}\right) \frac{b}{B_{0}}+\frac{D_{3}(r, \theta)}{\kappa}\right\} \dot{T}=0, \\
& \bar{P}_{x x}^{\prime}-\left(\frac{a}{A_{0}}\right)^{\prime}\left(P_{x x 0}+\mu_{0}\right) T+\left(\bar{\mu}+\bar{P}_{x x}\right) \frac{A_{0}^{\prime}}{A_{0}}-T\left(\frac{c}{C_{0}}\right)^{\prime}\left(P_{z z 0}-P_{x x 0}\right) \\
& -\left(\bar{P}_{z z}-\bar{P}_{x x}\right) \frac{C_{0}^{\prime}}{C_{0}}-T\left(\frac{b}{B_{0}}\right)^{\prime}\left(P_{y y 0}-P_{x x 0}\right)-\frac{B_{0}^{\prime}}{B_{0}}\left(\bar{P}_{y y}-\bar{P}_{x x}\right)+\frac{1}{r}\left(\bar{P}_{x y}^{\theta}\right. \\
& \left.-\bar{P}_{y y}+\bar{P}_{x x}\right)+T\left(\frac{c}{C_{0}}+\frac{2 b}{B_{0}}+\frac{a}{A_{0}}\right)^{\theta} \frac{P_{x y 0}}{r}+\frac{\bar{P}_{x y}}{r}\left(\frac{C_{0}^{\theta}}{C_{0}}+\frac{2 B_{0}^{\theta}}{B_{0}}+\frac{A_{0}^{\theta}}{A_{0}}\right) \\
& +\frac{P_{1}(t, r, \theta)}{\kappa}=0, \\
& \bar{P}_{y y}^{\theta}+\left(\bar{P}_{y y}+\bar{\mu}\right) \frac{A_{0}^{\theta}}{A_{0}}-T\left(\frac{a}{A_{0}}\right)^{\theta}\left(P_{y y 0}+\mu_{0}\right)-\left(\bar{P}_{x x}-\bar{P}_{y y}\right) \frac{B_{0}^{\theta}}{B_{0}} \\
& +T\left(\frac{b}{B_{0}}\right)^{\theta}\left(P_{x x 0}-P_{y y 0}\right)-\left(\bar{P}_{z z}-\bar{P}_{y y}\right) \frac{C_{0}^{\theta}}{C_{0}}+T\left(\frac{c}{C_{0}}\right)^{\theta}\left(P_{z z 0}-P_{y y 0}\right) \\
& +r T P_{x y 0}\left(\frac{c}{C_{0}}+\frac{2 b}{B_{0}}+\frac{a}{A_{0}}\right)^{\prime}+r\left(\frac{C_{0}^{\prime}}{C_{0}}+\frac{2}{r}+\frac{2 B_{0}^{\prime}}{B_{0}}+\frac{A_{0}^{\prime}}{A_{0}}\right) \bar{P}_{x y} \\
& +r \bar{P}_{x y}+\frac{D_{5}(t, r, \theta)}{\kappa}=0 .
\end{aligned}
$$

The perturbed part of the Ricci scalar becomes

$$
\begin{aligned}
& T e=\frac{2 T}{B_{0}^{2}}\left[\frac{C_{0}^{\prime} A_{0}^{\prime}}{C_{0} A_{0}}\left(\frac{a^{\prime}}{A_{0}^{\prime}}-\frac{a}{A_{0}}+\frac{c^{\prime}}{C_{0}^{\prime}}-\frac{c}{C_{0}}\right)+\left(\frac{a^{\prime \prime}}{A_{0}^{\prime \prime}}-\frac{a}{A_{0}}\right) \frac{A_{0}^{\prime \prime}}{A_{0}}+\left(\frac{b^{\prime \prime}}{B_{0}^{\prime \prime}}-\frac{b}{B_{0}}\right)\right. \\
& \times \frac{B_{0}^{\prime \prime}}{B_{0}}-\frac{1}{r}\left(\frac{a}{A_{0}}-\frac{c}{C_{0}}-\frac{b}{B_{0}}\right)^{\prime}+\left(\frac{\bar{c}^{\prime \prime}}{C_{0}^{\prime \prime}}-\frac{\bar{c}}{C_{0}}\right) \frac{C_{0}^{\prime \prime}}{C_{0}}-2\left(\frac{b}{B_{0}}\right)^{\prime} \frac{B_{0}^{\prime}}{B_{0}}+\left(\frac{b}{B_{0}}\right)^{\theta} \frac{B_{0}^{\theta}}{B_{0}} \\
& \times \frac{2}{r^{2}}+\left(\frac{c^{\theta \theta}}{C_{0}^{\theta \theta}}-\frac{c}{C_{0}}\right) \frac{C_{0}^{\theta \theta}}{C_{0}}+\left(\frac{b^{\theta \theta}}{B_{0}^{\theta \theta}}-\frac{b}{B_{0}}\right) \frac{B_{0}^{\theta \theta}}{B_{0}}+\left(\frac{a^{\theta \theta}}{A_{0}^{\theta \theta}}-\frac{a}{A_{0}}\right) \frac{A_{0}^{\theta \theta}}{A_{0}}+\frac{C_{0}^{\theta} A_{0}^{\theta}}{C_{0} A_{0}} \\
& \left.\times\left(\frac{a^{\theta}}{A_{0}^{\theta}}-\frac{a}{A_{0}}+\frac{c^{\theta}}{C_{0}^{\theta}}-\frac{c}{C_{0}}\right)\right]+\frac{2 \ddot{T}}{A_{0}^{2}}\left(\frac{c}{C_{0}}-\frac{b}{B_{0}}\right)-2 T b \frac{R_{0}}{B_{0}},
\end{aligned}
$$

which can be written as

$$
\ddot{T}(t)-\delta^{2}(r, \theta) T(t)=0,
$$


where $\delta^{2}$ is given in Appendix A. The solutions of Eq.(41) corresponds to the unstable as well as stable matter distributions. Since we are aiming to find the unstable range of collapsing body, so we assume $T(-\infty)=0$. For this purpose, we have

$$
T(t)=-\exp (\delta t)
$$

where $\delta^{2}>0$.

We can develop a relation between perturbed pressure components $\bar{P}_{i}$ and energy density $\bar{\mu}$ through the equation of state as (Harrison et al. 1965)

$$
\bar{P}_{i}=\Gamma_{1} \frac{P_{i 0}}{\mu_{0}+P_{i 0}} \bar{\mu},
$$

where $\Gamma_{1}$ is the adiabatic index which is taken as a constant identity throughout the paper. Using Eqs.(37) and (43), it follows that

$$
\begin{aligned}
& \bar{P}_{x x}=\frac{-\Gamma_{1}}{\mu_{0}+P_{x x 0}}\left[\frac{c}{C_{0}}\left(P_{z z 0}+\mu_{0}\right)+\left(P_{x x 0}+2 \mu_{0}+P_{y y 0}\right) \frac{b}{B_{0}}+\frac{D_{3}}{\kappa}\right] P_{x x 0} T, \\
& \bar{P}_{x y}=\frac{-\Gamma_{1}}{\mu_{0}+P_{x y 0}}\left[\frac{c}{C_{0}}\left(P_{z z 0}+\mu_{0}\right)+\left(P_{x x 0}+2 \mu_{0}+P_{y y 0}\right) \frac{b}{B_{0}}+\frac{D_{3}}{\kappa}\right] P_{x y 0} T, \\
& \bar{P}_{y y}=\frac{-\Gamma_{1}}{\mu_{0}+P_{y y 0}}\left[\frac{c}{C_{0}}\left(P_{z z 0}+\mu_{0}\right)+\left(P_{x x 0}+2 \mu_{0}+P_{y y 0}\right) \frac{b}{B_{0}}+\frac{D_{3}}{\kappa}\right] P_{y y 0} T, \\
& \bar{P}_{z z}=\frac{-\Gamma_{1}}{\mu_{0}+P_{z z 0}}\left[\frac{c}{C_{0}}\left(P_{z z 0}+\mu_{0}\right)+\left(P_{x x 0}+2 \mu_{0}+P_{y y 0}\right) \frac{b}{B_{0}}+\frac{D_{3}}{\kappa}\right] P_{z z 0} T .
\end{aligned}
$$

Using these equations in Eq.(38), we obtain the collapse equation as follows

$$
\begin{aligned}
& -T \Gamma_{1}\left[\left\{\left(P_{z z 0}+\mu_{0}\right) \frac{c}{C_{0}}+\left(P_{y y 0}+P_{x x 0}\right) \frac{b}{B_{0}}+\frac{D_{3}}{\kappa}\right\}\left(\frac{P_{x x 0}}{\mu_{0}+P_{x x 0}}\right)\right]^{\prime} \\
& +T\left(\frac{c}{C_{0}}\right)^{\prime}\left(P_{x x 0}-P_{z z 0}\right)+T\left\{\Gamma_{1} \frac{P_{x x 0}}{P_{x x 0}+\mu_{0}}+1\right\} \frac{A_{0}^{\prime}}{A_{0}}\left[-\frac{c}{C_{0}}\left(P_{z z 0}+\mu_{0}\right)\right. \\
& \left.-\left(P_{y y 0}+P_{x x 0}+2 \mu_{0}\right) \frac{b}{B_{0}}-\frac{D_{3}}{\kappa}\right]-T\left(\frac{a}{A_{0}}\right)^{\prime}\left(\mu_{0}+P_{x x 0}\right)+T\left(\frac{b}{B_{0}}\right)^{\prime} \\
& \times\left(P_{x x 0}-P_{y y 0}\right)+\Gamma_{1} T \frac{C_{0}^{\prime}}{C_{0}}\left[\frac{c}{C_{0}}\left\{P_{z z 0}-\frac{\left(P_{z z 0}+\mu_{0}\right) P_{x x 0}}{\left(P_{z z 0}+\mu_{0}\right)}\right\}+\left(P_{x x 0}+2 \mu_{0}\right.\right. \\
& \left.+P_{y y 0}\right)\left\{\frac{P_{z z 0}}{\mu_{0}+P_{z z 0}}-\frac{P_{x x 0}}{P_{x x 0}+\mu_{0}}\right\} \frac{b}{B_{0}}+\left\{\frac{P_{z z 0}}{\mu_{0}+P_{z z 0}}-\frac{P_{x x 0}}{P_{x x 0}+\mu_{0}}\right\} \\
& \left.\times \frac{D_{3}}{\kappa}\right]+\left(\frac{1}{r}+\frac{B_{0}^{\prime}}{B_{0}}\right) \Gamma_{1} T\left(\frac{P_{y y 0}}{P_{y y 0}+\mu_{0}}-\frac{P_{x x 0}}{P_{x x 0}+\mu_{0}}\right)\left[\frac{c}{C_{0}}\left(P_{z z 0}+\mu_{0}\right)\right. \\
& \left.+\left(P_{x x 0}+P_{y y 0}+2 \mu_{0}\right) \frac{b}{B_{0}}+\frac{D_{3}}{\kappa}\right]-\Gamma_{1} \frac{T}{r}\left[\left\{\frac{c}{C_{0}}\left(P_{z z 0}+\mu_{0}\right)+\left(P_{x x 0}\right.\right.\right.
\end{aligned}
$$




$$
\begin{aligned}
& \left.\left.\left.+P_{y y 0}+2 \mu_{0}\right) \frac{b}{B_{0}}+\frac{D_{3}}{\kappa}\right\} \frac{P_{x y 0}}{\left(P_{x y 0}+\mu_{0}\right)}\right]^{\theta}-\Gamma_{1} \frac{T}{r}\left(\frac{A_{0}^{\theta}}{A_{0}}+\frac{2 B_{0}^{\theta}}{B_{0}}+\frac{C_{0}^{\theta}}{C_{0}}\right) \\
& {\left[\left\{\frac{c}{C_{0}}\left(P_{z z 0}+\mu_{0}\right)+\left(P_{x x 0}+P_{y y 0}+2 \mu_{0}\right) \frac{b}{B_{0}}+\frac{D_{3}}{\kappa}\right\} \frac{P_{x y 0}}{\left(P_{x y 0}+\mu_{0}\right)}\right]} \\
& +P_{x y 0} \frac{T}{r}\left(\frac{c}{C_{0}}+\frac{b}{B_{0}}+\frac{a}{A_{0}}\right)^{\theta}+\frac{T(t) D_{4}(r, \theta)}{\kappa}=0,
\end{aligned}
$$

where $P_{1}=T(t) D_{4}(r, \theta)$ ((obtained by using Eq.(42) $)$. This equation has fundamental importance in stellar instability analysis of the collapsing astronomical axial body in $f(R)$ gravity.

\section{Instability Regions}

In this section, we investigate the instability regions of a system at both $\mathrm{N}$ and $\mathrm{pN}$ approximations in $f(R)$ gravity. We also find the importance of adiabatic index $\Gamma_{1}$ in this framework.

\subsection{Newtonian Approximation}

Here, we consider the $\mathrm{N}$ limits as $A_{0}=1, B_{0}=1$, for the investigation of instability ranges in the $\mathrm{N}$ era. Moreover, we assume that $P_{x x 0}<0$ indicates collapsing fluid and $C_{0}=r$

as the radial coordinate. Under these assumptions, the collapse equation (44) turns out to be

$$
\begin{aligned}
& -\Gamma_{1} T\left[\left(\frac{c}{r}+2 b\right) P_{x x 0}\right]^{\prime}+\Gamma_{1} \frac{T}{r}\left[\left(\frac{c}{r}+2 b\right)\left(P_{y y 0}-P_{x x 0}\right)\right]+\Gamma_{1} T\left[\left(\frac{c}{r}+2 b\right)\right. \\
& \left.\times\left(P_{z z 0}-P_{x x 0}\right)\right]-\frac{\Gamma_{1} T}{r}\left[\left(\frac{c}{r}+2 b\right) P_{x y 0}\right]^{\theta}=a^{\prime} \mu_{0}+T\left(\frac{c}{r}\right)^{\prime}\left(P_{z z 0}-P_{x x 0}\right) \\
& +T b^{\prime}\left(P_{y y 0}-P_{x x 0}\right)-\frac{T}{r}\left(a+b+\frac{c}{r}\right)^{\theta} P_{x y 0}-\frac{D_{4\left(N_{0}\right)}}{\kappa},
\end{aligned}
$$

where $D_{4\left(N_{0}\right)}$ represents terms of $D_{4}$ under $\mathrm{N}$ limit with $C_{0}=r$. The required dynamical instability range for the collapsing axial symmetric body is given using Eq.(42) as

$$
\Gamma_{1}<\frac{a^{\prime} \mu_{0}+\left(P_{z z 0}-P_{x x 0}\right)\left(\frac{c}{r}\right)^{\prime}-\frac{D_{4\left(N_{0}\right)}}{\kappa}+\Omega_{1}(r)}{\frac{1}{r}\left(\frac{c}{r}+2 b\right)\left(P_{y y 0}-2 P_{x x 0}+P_{z z 0}\right)+\Omega_{2}(r)},
$$

where we defined

$$
\Omega_{1}=b^{\prime}\left(P_{y y 0}-P_{x x 0}\right)-\frac{P_{x y 0}}{r}\left(a+b+\frac{c}{r}\right)^{\theta}
$$




$$
\Omega_{2}=-\frac{1}{r}\left[\left(\frac{c}{r}+2 b\right) P_{x x 0}\right]^{\theta}-\left[\left(\frac{c}{r}+2 b\right) P_{x x 0}\right]^{\prime} .
$$

This suggests that $\Gamma_{1}$ plays a key role in the investigation of stability at $\mathrm{N}$ approximation. Moreover, the fluid distribution will be unstable unless (46) is satisfied. Under the constant Ricci scalar condition, i.e., $R=\tilde{R}$ and $e=\tilde{e}=$ constant, we have

$$
\Gamma_{1}<\frac{a^{\prime} \mu_{0}+\left(P_{z z 0}-P_{x x 0}\right)\left(\frac{c}{r}\right)^{\prime}+\left(2 \delta_{(N)}^{2}-\tilde{R}\right) \frac{\epsilon \tilde{e}}{\kappa}+\Omega_{1}}{\frac{1}{r}\left(\frac{c}{r}+2 b\right)\left(P_{y y 0}-2 P_{x x 0}+P_{z z 0}\right)+\Omega_{2}} .
$$

For $\epsilon \rightarrow 0$, we get the same inequality as above lest $\zeta=0$ which corresponds to GR solution (Sharif and Bhatti 2013b).

\subsection{Post-Newtonian Approximation}

For the dynamical range of instability in the $\mathrm{pN}$ limit, we assume

$$
A_{0}=1-\frac{m_{0}}{r}, \quad B_{0}=1+\frac{m_{0}}{r},
$$

and take the effects upto $O\left(\frac{m_{0}}{r}\right)$. Using these quantities in the collapse equation (44), we get the instability range as

$$
\Gamma_{1}<\frac{\left(1+\frac{m_{0}}{r}\right)\left(1-\frac{m_{0}}{r}\right)^{\prime} \zeta_{1}+\Omega_{3}-\frac{D_{4\left(p N_{0}\right)}}{\kappa}}{-\frac{\zeta_{1}}{r}\left[\left(1-\frac{m_{0}}{r}\right)^{\theta}\left(1+\frac{m_{0}}{r}\right) \frac{P_{x y 0}}{P_{x y 0}+\mu_{0}}\right]+\Omega_{4}}
$$

where

$$
\begin{aligned}
& \zeta_{1}=\frac{c}{r}\left(P_{z z 0}+\mu_{0}\right)+\left(P_{x x 0}+P_{y y 0}+2 \mu_{0}\right) b\left(1-\frac{m_{0}}{r}\right)+\frac{D_{3 p N_{0}}}{\kappa}, \\
& \Omega_{1}=\left(b-\frac{b m_{0}}{r}\right)^{\prime}\left(P_{y y 0}-P_{x x 0}\right)-\frac{P_{x y 0}}{r}\left[a+b+\frac{c}{r}+\frac{m_{0}}{r}(a+b)\right]^{\theta} \\
& +\left(a-\frac{a m_{0}}{r}\right)^{\prime}\left(P_{x x 0}+\mu_{0}\right), \\
& \Omega_{2}=-\left(1+\frac{m_{0}}{r}\right)\left(1-\frac{m_{0}}{r}\right)^{\prime} \frac{P_{x x 0} \zeta_{1}}{P_{x x 0}+\mu_{0}}+\left\{\frac{1}{r}+\left(1+\frac{m_{0}}{r}\right)^{\prime}\left(1-\frac{m_{0}}{r}\right)\right\} \\
& \times\left(\frac{\zeta_{1} P_{y y 0}}{P_{y y 0}+\mu_{0}}-\frac{\zeta_{1} P_{x x 0}}{P_{x x 0}+\mu_{0}}\right)+\frac{c}{r^{2}}\left\{P_{z z 0}-\frac{\left(P_{z z 0}+\mu_{0}\right) P_{x x 0}}{P_{x x 0}+\mu_{0}}\right\}+\frac{1}{r}[(b \\
& \left.\left.\left.-\frac{b m_{0}}{r}\right)_{\left(2 \mu_{0}\right.}+P_{x x 0}+P_{y y 0}\right)+\frac{D_{3}}{\kappa}\right]\left(\frac{P_{z z 0}}{P_{z z 0}+\mu_{0}}-\frac{P_{x x 0}}{P_{x x 0}+\mu_{0}}\right) \\
& -\left(\frac{P_{x x 0 \zeta_{1}}}{P_{x x 0}+\mu_{0}}\right)_{, 1}-\left(\frac{P_{x y 0 \zeta_{1}}}{P_{x y 0}+\mu_{0}}\right)_{, 0}-\frac{\zeta_{1}}{r}\left[2\left(1-\frac{m_{0}}{r}\right)\left(1+\frac{m_{0}}{r}\right)^{\theta} \frac{P_{x y 0}}{P_{x y 0}+\mu_{0}}\right],
\end{aligned}
$$


where $D_{3 p N_{0}}$ and $D_{4 p N_{0}}$ correspond to those quantities of $D_{3}$ and $D_{4}$ that are computed under pN limits (mentioned in Eq.(48)) with $C_{0}=r$, respectively. Under constant curvature condition, we obtain the same inequality as (49) with the difference that $D_{3 p N_{0}}$ and $D_{4 p N_{0}}$ reduces to $\zeta_{1}$ and $\zeta_{2}$, respectively and are given as

$$
\begin{aligned}
\zeta_{1} & =-\epsilon \tilde{e} \tilde{R}-2 \epsilon \tilde{e}\left(1-\frac{m_{0}}{r}\right)^{\prime}\left(1-\frac{m_{0}}{r}\right)\left[3\left(1-\frac{m_{0}}{r}\right)^{\prime}\left(1+\frac{m_{0}}{r}\right)\right. \\
& \left.-2\left(1+\frac{m_{0}}{r}\right)\left(1-\frac{m_{0}}{r}\right)+\frac{2}{r}\right]-\frac{2 \epsilon \tilde{e}}{r^{2}}\left(1-\frac{m_{0}}{r}\right)^{\theta}\left(1-\frac{m_{0}}{r}\right) \\
& \times\left[3\left(1-\frac{m_{0}}{r}\right)^{\theta}\left(1+\frac{m_{0}}{r}\right)-2\left(1+\frac{m_{0}}{r}\right)^{\theta}\left(1-\frac{m_{0}}{r}\right)\right], \\
\zeta_{2} & =\frac{\epsilon \tilde{e}}{\kappa}\left[2 \delta^{2}\left(1+\frac{m_{0}}{r}\right)+2 \delta^{2}\left(1-\frac{m_{0}}{r}\right)^{\prime}\left(1+\frac{3 m_{0}}{r}\right)\right] .
\end{aligned}
$$

For $\epsilon \rightarrow 0$, we obtain the instability constraint given in (49) with $\zeta_{1}=\zeta_{2}=0$ This indicates that our results exactly coincide with GR solution (Sharif and Bhatti 2013b).

\section{Conclusions}

In this work, we have explored factors which affect the gravitational collapse of nonstatic axial matter distribution in $f(R)$ gravity. Our analysis provides corrections to the usual GR field equations thus modify the dynamical evolutionary phases of collapse process. The field equations and the dynamical equations are formulated that are perturbed through the perturbation scheme. The collapse equation is then constructed from the dynamical equations to discuss the instability range by assuming the relationship of perturbed pressure and energy density through equation of state.

It is well-known that instability issue of stellar bodies are explored by following two techniques. First scheme is based on numerical methods which assists us to interpret realistic collapsing scenarios. Nevertheless, these results are generally restricted and depends upon model under consideration. The second scheme yields analytical solutions, which are relatively easy to examine and provides useful results in the theory of structure formation of stellar systems. In this paper, we have used analytical approach to examine the instability regions at $\mathrm{N}$ and $\mathrm{pN}$ eras.

We have found the instability ranges for $\mathrm{N}$ and $\mathrm{pN}$ regimes. For $\mathrm{N}$ regime, the system will be unstable if it satisfies the inequality (46) while for $\mathrm{pN}$ regime, it will remain unstable if the inequality (49) is satisfied. The violation of these inequalities will lead to stable configuration of the axial symmetry. These constraints indicate that stability of the collapsing anisotropic 
axial astronomical matter is controlled by the radial profile of pressure anisotropy, energy density and the dark source $f(R)$ terms. The relations (46) and (49) are quoted in terms of the adiabatic index $\Gamma_{1}$ that indicate its importance and compatibility with Chandrasekhar (1964). We see that the adiabatic index depends upon the physical parameters of the fluid distribution. It is worth mentioning here that anisotropic pressure disturbs the stability of the axial symmetry and makes the system more unstable as the evolution proceeds.

It is well-known that the stability of self-gravitating systems has a direct correspondence with hydrostatic equilibrium conditions. Equations that describe such equilibrium phases are related to $f(R)$ field equations which couple the fluid distribution with its gravitational field. The dark source terms that originate due to $f(R)$ model (Eq.(17)) in the field equations decrease the range of instability thus lags the $\mathrm{BH}$ formation. It is seen from relations (46) and (49) that extra-order $f(R)$ corrections, i.e., $\epsilon$ terms extend the stability ranges of stellar structures which is in agreement with Farinelli et al. (2014). Moreover, it is also wellestablished that the inflationary candidate $f(R)=R+\epsilon R^{2}$ terms cause anti-evaporation of the Schwarzschild-de Sitter black hole (so called Nariai black hole) on classical level. Finally, all our results lead to GR solutions (Sharif and Bhatti 2013b) under the limit $\epsilon \rightarrow 0$.

\section{Appendix A}

The higher curvature terms for Eqs.(14)-(16) are given as

$$
\begin{aligned}
& D_{0}=\frac{1}{A}\left[\frac{f-R f_{R}}{2}+\frac{f_{R}^{\prime \prime}}{B^{2}}-\frac{\dot{f_{R}}}{A^{2}}\left(\frac{2 \dot{B}}{B}+\frac{\dot{C}}{C}\right)+\frac{f_{R}^{\prime}}{B^{2}}\left(\frac{C^{\prime}}{C}-\frac{2 B^{\prime}}{B}+\frac{1}{r}\right)\right. \\
& \left.+\frac{f_{R}^{\theta}}{B^{2} r^{2}}\left(\frac{C^{\theta}}{C}-2 \frac{B^{\theta}}{B}\right)+\frac{f_{R}^{\theta \theta}}{B^{2} r^{2}}\right]_{, 0}-\frac{1}{A B^{2}}\left(\dot{f}_{R}^{\prime}-\frac{A^{\prime}}{A} \dot{f}_{R}-\frac{\dot{B}}{B} f_{R}^{\prime}\right) \\
& \times\left(3 \frac{A^{\prime}}{A}+2 \frac{B^{\prime}}{B}+\frac{C^{\prime}}{C}+\frac{1}{r}\right)+\frac{1}{A B^{2} r^{2}}\left(\frac{3 A^{\theta}}{A}+\frac{2 B^{\theta}}{B}+\frac{C^{\theta}}{C}\right) \\
& \times\left(\dot{f}_{R}^{\theta}-\frac{A^{\theta}}{A} \dot{f}_{R}-\frac{\dot{B}}{B} f_{R}^{\theta}\right)+A\left[\frac{(-1)}{A^{2} B^{2}}\left(\dot{f}_{R}^{\prime}-\frac{A^{\prime}}{A} \dot{f}_{R}-\frac{\dot{B}}{B} f_{R}^{\prime}\right)\right]_{, 1} \\
& +A\left[\frac{-1}{A^{2} B^{2} r^{2}}\left(\dot{f}_{R}^{\theta}-\frac{A^{\theta}}{A} \dot{f}_{R}-\frac{\dot{B}}{B} f_{R}^{\theta}\right)\right]_{, 2}+\frac{\dot{C}}{A C}\left[\frac{f_{R}^{\prime}}{B^{2}}\left(\frac{C^{\prime}}{C}+\frac{2}{r}-\frac{A^{\prime}}{A}\right)\right. \\
& \left.-\frac{f_{R}^{\theta}}{B^{2} r^{2}}\left(\frac{C^{\theta}}{C}-\frac{A^{\theta}}{A}\right)-\frac{\dot{f_{R}}}{A^{2}}\left(\frac{\dot{A}}{A}+\frac{\dot{C}}{C}\right)\right]+\frac{\dot{B}}{A B}\left[\frac{f_{R}^{\prime \prime}}{B^{2}}+\frac{f_{R}^{\theta \theta}}{B^{2} r^{2}}+\frac{2 \ddot{f}_{R}}{A^{2}}\right.
\end{aligned}
$$




$$
\begin{aligned}
& \left.-\frac{f_{R}^{\theta}}{B^{2} r^{2}}\left(\frac{A^{\theta}}{A}\right)-\frac{2 \dot{f}_{R}}{A^{2}}\left(\frac{\dot{A}}{A}+\frac{\dot{B}}{B}+\frac{\dot{C}}{C}\right)-\frac{f_{R}^{\prime}}{B^{2}}\left(\frac{2 A^{\prime}}{A}+\frac{2 B^{\prime}}{B}+\frac{3}{r}\right)\right], \\
& D_{1}=\frac{(-1)}{B}\left[\frac{f-R f_{R}}{2}-\frac{\ddot{f_{R}}}{A^{2}}+\frac{f_{R}^{\theta \theta}}{B^{2} r^{2}}+\frac{f_{R}^{\prime}}{B^{2}}\left(\frac{A^{\prime}}{A}-\frac{B^{\prime}}{B}-\frac{1}{r}+\frac{C^{\prime}}{C}\right)\right. \\
& \left.+\frac{\dot{f_{R}}}{A^{2}}\left(\frac{\dot{A}}{A}-\frac{\dot{B}}{B}+\frac{\dot{C}}{C}\right)+\frac{f_{R}^{\theta}}{B^{2} r^{2}}\left(\frac{A^{\theta}}{A}-3 \frac{B^{\theta}}{B}+\frac{C^{\theta}}{C}\right)\right]_{, 1}-\frac{1}{A^{2} B} \\
& \times\left(\dot{f}_{R}^{\prime}-\frac{A^{\prime}}{A} \dot{f}_{R}-\frac{\dot{B}}{B} f_{R}^{\prime}\right)\left(\frac{\dot{A}}{A}+4 \frac{\dot{B}}{B}+\frac{\dot{C}}{C}\right)+\frac{1}{B^{3} r^{2}}\left(\frac{A^{\theta}}{A}+\frac{4 B^{\theta}}{B}\right) \\
& \times\left(f_{R}^{\prime \theta}-\frac{B^{\theta}}{B} f_{R}^{\prime}-\frac{f_{R}^{\theta}}{r}-\frac{B^{\prime}}{B} f_{R}^{\theta}\right)+B\left[\frac{(-1)}{A^{2} B^{2}}\left(\dot{f}_{R}^{\prime}-\frac{A^{\prime}}{A} \dot{f}_{R}-\frac{\dot{B}}{B} f_{R}^{\prime}\right)\right]_{, 0} \\
& +B\left[\frac{1}{B^{4} r^{2}}\left(f_{R}^{\prime \theta}-\frac{B^{\theta}}{B} f_{R}^{\prime}-\frac{f_{R}^{\theta}}{r}-\frac{B^{\prime}}{B} f_{R}^{\theta}\right)\right]_{, 2}+\frac{A^{\prime}}{A B}\left[\frac{f_{R}^{\prime \prime}}{B^{2}}+\frac{\ddot{f}_{R}}{A^{2}}\right. \\
& -\frac{f_{R}^{\theta \theta}}{B^{2} r^{2}}-\frac{\dot{f_{R}}}{A^{2}}\left(\frac{\dot{B}}{B}+\frac{\dot{A}}{A}+2 \frac{\dot{C}}{C}\right)+\frac{f_{R}^{\theta}}{B^{2} r^{2}}\left(\frac{B^{\theta}}{B}-\frac{A^{\theta}}{A}\right)+\frac{f_{R}^{\prime}}{B^{2}}\left(\frac{2}{r}\right. \\
& \left.\left.-\frac{B^{\prime}}{B}-\frac{A^{\prime}}{A}\right)\right]+\left(\frac{1}{r}+\frac{B^{\prime}}{B}\right) \frac{1}{B}\left[\frac{f_{R}^{\prime \prime}}{B^{2}}-\frac{f_{R}^{\theta \theta}}{B^{2} r^{2}}-\frac{2 \dot{f_{R}} \dot{C}}{A^{2} C}-\frac{f_{R}^{\prime}}{B^{2} r}\right. \\
& \left.+\frac{2 B^{\theta} f_{R}^{\theta}}{B^{3} r^{2}}\right]-\frac{C^{\prime}}{B C}\left[\frac{f_{R}^{\theta}}{B^{2} r^{2}}\left(\frac{B^{\theta}}{B}-\frac{C^{\theta}}{C}\right)-\frac{\dot{f_{R}}}{A^{2}}\left(\frac{\dot{B}}{B}+\frac{\dot{C}}{C}\right)\right. \\
& \left.-\frac{f_{R}^{\prime}}{B^{2}}\left(\frac{B^{\prime}}{B}+\frac{C^{\prime}}{C}\right)+\frac{f_{R}^{\prime \prime}}{B^{2}}\right] \\
& D_{2}=B\left[\frac{(-1)}{A^{2} B^{2} r^{2}}\left(\dot{f}_{R}^{\theta}-\frac{A^{\theta}}{A} \dot{f}_{R}-\frac{\dot{B}}{B} f_{R}^{\theta}\right)\right]_{, 0}+B^{2}\left[\frac { 1 } { B ^ { 4 } r ^ { 2 } } \left(f_{R}^{\prime \theta}-\frac{B^{\theta}}{B} f_{R}^{\prime}\right.\right. \\
& \left.\left.-\frac{f_{R}^{\theta}}{r}-\frac{B^{\prime}}{B} f_{R}^{\theta}\right)\right]_{, 1}-\frac{1}{A^{2} r^{2}}\left(\dot{f}_{R}^{\theta}-\frac{A^{\theta}}{A} \dot{f_{R}}-\frac{\dot{B}}{B} f_{R}^{\theta}\right)\left(\frac{3 \dot{B}}{B}+\frac{\dot{C}}{C}\right) \\
& +\frac{1}{B^{2} r^{2}}\left(f_{R}^{\prime \theta}-\frac{B^{\theta}}{B} f_{R}^{\prime}-\frac{f_{R}^{\theta}}{r}-\frac{B^{\prime}}{B} f_{R}^{\theta}\right)\left(\frac{A^{\prime}}{A}+\frac{3}{r}+\frac{4 B^{\prime}}{B}+\frac{C^{\prime}}{C}\right) \\
& +\frac{A^{\theta}}{A r^{2}}\left[\frac{f^{\theta \theta}}{B^{2} r^{2}}+\frac{\ddot{f}_{R}}{A^{2}}-\frac{\dot{f_{R}}}{A^{2}}\left(\frac{\dot{A}}{A}+\frac{\dot{B}}{B}\right)+\frac{f_{R}^{\prime}}{B^{2}}\left(\frac{1}{r}-\frac{B^{\prime}}{B}-\frac{A^{\prime}}{A}\right)\right. \\
& \left.-\frac{f_{R}^{\theta}}{B^{2} r^{2}}\left(\frac{A^{\theta}}{A}+\frac{B^{\theta}}{B}\right)\right]+\frac{B^{\theta}}{B r^{2}}\left[\frac{f_{R}^{\theta \theta}}{B^{2} r^{2}}-\frac{f_{R}^{\prime \prime}}{B^{2}}-\frac{f_{R}^{\prime}}{B^{2} r}+\frac{\dot{f}_{R}}{A^{2}}\left(\frac{2 \dot{C}}{C}\right)\right.
\end{aligned}
$$




$$
\begin{aligned}
& \left.-\frac{f_{R}^{\theta}}{B^{2} r^{2}}\left(\frac{2 B^{\theta}}{B}\right)\right]-\frac{1}{r^{2}}\left[\frac{f-R f_{R}}{2}-\frac{\ddot{f_{R}}}{A^{2}}+\frac{f_{R}^{\prime \prime}}{B^{2}}+\frac{\dot{f_{R}}}{A^{2}}\left(\frac{\dot{A}}{A}-\frac{\dot{B}}{B}-\frac{\dot{C}}{C}\right)\right. \\
& \left.+\frac{f_{R}^{\prime}}{B^{2}}\left(\frac{A^{\prime}}{A}+\frac{C^{\prime}}{C}-\frac{B^{\prime}}{B}\right)+\frac{f_{R}^{\theta}}{B^{2} r^{2}}\left(\frac{A^{\theta}}{A}+\frac{C^{\theta}}{C}-\frac{B^{\theta}}{B}\right)\right]_{, 2}+\frac{C^{\theta}}{C r^{2}}\left[\frac{f_{R}^{\theta \theta}}{B^{2} r^{2}}\right. \\
& \left.+\frac{\dot{f}_{R}}{A^{2}}\left(\frac{\dot{C}}{C}-\frac{\dot{B}}{B}\right)-\frac{f_{R}^{\prime}}{B^{2}}\left(\frac{B^{\prime}}{B}+\frac{C^{\prime}}{C}+\frac{1}{r}\right)-\frac{f_{R}^{\theta}}{B^{2} r^{2}}\left(\frac{B^{\theta}}{B}+\frac{C^{\theta}}{C}\right)\right] .
\end{aligned}
$$

The static portions of Eqs. (35) and (36) are computed as

$$
\begin{aligned}
& D_{1 S}=\frac{-2 \epsilon}{B_{0}}\left[-\frac{R_{0}^{2}}{4}+\frac{R_{0}^{\theta \theta}}{B_{0}^{2} r^{2}}+\frac{R_{0}^{\prime}}{B_{0}^{2}}\left(\frac{A_{0}^{\prime}}{A_{0}}-\frac{B_{0}^{\prime}}{B_{0}}-\frac{1}{r}+\frac{C_{0}^{\prime}}{C_{0}}\right)+\frac{R_{0}^{\theta}}{B_{0}^{2} r^{2}}\right. \\
& \left.\times\left(\frac{A_{0}^{\theta}}{A_{0}}-3 \frac{B_{0}^{\theta}}{B_{0}}+\frac{C_{0}^{\theta}}{C_{0}}\right)\right]_{, 1}+\frac{2 \epsilon}{B_{0}^{3} r^{2}}\left(\frac{A_{0}^{\theta}}{A_{0}}+\frac{4 B_{0}^{\theta}}{B_{0}}\right)\left(R_{0}^{\theta}-R_{0} \frac{B_{0}^{\theta}}{B_{0}}\right. \\
& \left.-\frac{R_{0}^{\theta}}{r}-R_{0}^{\theta} \frac{B_{0}^{\prime}}{B_{0}}\right)+2 \epsilon B_{0}\left[R_{0}^{\prime \theta}-\frac{B_{0}^{\theta}}{B_{0}} R_{0}-\frac{R_{0}^{\theta}}{r}-R_{0}^{\theta} \frac{B_{0}^{\prime}}{B_{0}}\right]_{, 2}+\frac{2 \epsilon A_{0}^{\prime}}{A_{0} B_{0}^{3}} \\
& \times\left[R_{0}^{\prime \prime}-\frac{R_{0}^{\theta \theta}}{r^{2}}+\frac{R_{0}^{\theta}}{r^{2}}\left(\frac{B_{0}^{\theta}}{B_{0}}-\frac{A_{0}^{\theta}}{A_{0}}\right)+R_{0}^{\prime}\left(\frac{2}{r}-\frac{B_{0}^{\prime}}{B_{0}}-\frac{A_{0}^{\prime}}{A_{0}}\right)\right]+\frac{2 \epsilon}{B_{0}^{2}} \\
& \times\left(\frac{1}{r}+\frac{B_{0}^{\prime}}{B_{0}}\right)\left[R_{0}^{\prime \prime}-\frac{R_{0}^{\theta \theta}}{r^{2}}-\frac{R_{0}^{\prime}}{r}+\frac{R_{0}^{\theta}}{r^{2}}\left(\frac{2 B_{0}^{\theta}}{B_{0}}\right)\right]-\frac{2 \epsilon C_{0}^{\prime}}{C_{0} B_{0}^{3}}\left[\frac { R _ { 0 } ^ { \theta } } { r ^ { 2 } } \left(\frac{B_{0}^{\theta}}{B_{0}}\right.\right. \\
& \left.\left.-\frac{C_{0}^{\theta}}{C_{0}}\right)-R_{0}^{\prime}\left(\frac{B_{0}^{\prime}}{B_{0}}+\frac{C_{0}^{\prime}}{C_{0}}\right)+R_{0}^{\prime \prime}\right] \text {, } \\
& D_{2 S}=B_{0}^{2}\left[\frac{2 \epsilon}{B_{0}^{4} r^{2}}\left\{R_{0}^{\prime \theta}-\frac{B_{0}^{\theta}}{B_{0}} R_{0}-\frac{R_{0}^{\theta}}{r}-R_{0}^{\theta} \frac{B_{0}^{\prime}}{B_{0}}\right\}\right]_{, 1}+\frac{2 \epsilon}{B_{0}^{2} r^{2}}\left(R_{0}^{\prime \theta}-\frac{B_{0}^{\theta}}{B_{0}} R_{0}\right. \\
& \left.-\frac{R_{0}^{\theta}}{r}-R_{0}^{\theta} \frac{B_{0}^{\prime}}{B_{0}}\right)\left(\frac{A_{0}^{\prime}}{A_{0}}+\frac{4 B_{0}^{\prime}}{B_{0}}+\frac{3}{r}+\frac{C_{0}^{\prime}}{C_{0}}\right)+\frac{2 \epsilon A_{0}^{\theta}}{r A_{0} B_{0}^{2}}\left[\frac{R_{0}^{\theta \theta}}{r^{2}}+R_{0}^{\prime}\left(\frac{1}{r}\right.\right. \\
& \left.\left.-\frac{B_{0}^{\prime}}{B_{0}}-\frac{A_{0}^{\prime}}{A_{0}}\right)-\frac{R_{0}^{\theta}}{r^{2}}\left(\frac{B_{0}^{\theta}}{B_{0}}+\frac{A_{0}^{\theta}}{A_{0}}\right)\right]+\frac{2 \epsilon B_{0}^{\theta}}{B_{0}^{3} r^{2}}\left[\frac{R_{0}^{\theta \theta}}{r^{2}}-R_{0}^{\prime \prime}-\frac{R_{0}^{\prime}}{r}-\frac{R_{0}^{\theta}}{r^{2}}\right. \\
& \left.\times\left(\frac{2 B_{0}^{\theta}}{B_{0}}\right)\right]-\frac{2 \epsilon}{B_{0}^{2} r^{2}}\left[-\frac{B_{0}^{2} R_{0}^{2}}{4}+R_{0}^{\prime \prime}+R_{0}^{\prime}\left(\frac{C_{0}^{\prime}}{C_{0}}-\frac{B_{0}^{\prime}}{B_{0}}+\frac{A_{0}^{\prime}}{A_{0}}\right)+\frac{R_{0}^{\theta}}{r^{2}}\right. \\
& \left.\times\left(\frac{A_{0}^{\theta}}{A_{0}}-\frac{B_{0}^{\theta}}{B_{0}}+\frac{C_{0}^{\theta}}{C_{0}}\right)\right]_{, 2}+\frac{2 \epsilon C_{0}^{\theta}}{C_{0} B_{0}^{2} r^{2}}\left[\frac{R_{0}^{\theta \theta}}{r^{2}}-R_{0}^{\prime}\left(\frac{1}{r}+\frac{B_{0}^{\prime}}{B_{0}}+\frac{C_{0}^{\prime}}{C_{0}}\right)\right. \\
& \left.-\frac{R_{0}^{\theta}}{r^{2}}\left(\frac{B_{0}^{\theta}}{B_{0}}+\frac{C_{0}^{\theta}}{C_{0}}\right)\right] \text {. }
\end{aligned}
$$

The perturbed portions of Eqs.(37)-(39) are

$$
D_{3}=-\epsilon e R_{0}+\frac{2 \epsilon}{B_{0}^{2}}\left(e^{\prime \prime}-\frac{2 b R_{0}^{\prime \prime}}{B_{0}}\right)+\frac{2 \epsilon}{B_{0}^{2} r^{2}}\left(e^{\theta \theta}-\frac{2 b R_{0}^{\theta \theta}}{B_{0}}\right)+\frac{2 \epsilon}{B_{0}^{2}}\left(e^{\prime}\right.
$$




$$
\begin{aligned}
& \left.-\frac{2 b R_{0}^{\prime}}{B_{0}}\right)\left(\frac{C_{0}^{\prime}}{C_{0}}-2 \frac{B_{0}^{\prime}}{B_{0}}+\frac{1}{r}\right)+\frac{2 \epsilon R_{0}^{\prime}}{B_{0}^{2}}\left\{\left(\frac{c}{C_{0}}\right)^{\prime}-2\left(\frac{b}{B_{0}}\right)^{\prime}\right\}+\frac{2 \epsilon}{B_{0}^{2} r^{2}} \\
& \times\left(e^{\theta}-\frac{2 b R_{0}^{\theta}}{B_{0}}\right)\left(\frac{C_{0}^{\theta}}{C_{0}}-2 \frac{B_{0}^{\theta}}{B_{0}}\right)+\frac{2 \epsilon R_{0}^{\theta}}{B^{2} r^{2}}\left\{\left(\frac{c}{C_{0}}\right)^{\theta}-2\left(\frac{b}{B_{0}}\right)^{\theta}\right\}+\frac{2 \epsilon}{B_{0}^{2}} \\
& \times\left(e^{\prime}-\frac{e A_{0}^{\prime}}{A_{0}}-b \frac{R_{0}^{\prime}}{B_{0}}\right)\left(3 \frac{A_{0}^{\prime}}{A_{0}}-2 \frac{B_{0}^{\prime}}{B_{0}}+\frac{1}{r}+\frac{C_{0}^{\prime}}{C_{0}}\right)-\frac{2 \epsilon}{B_{0}^{2} r^{2}}\left(e^{\theta}-\frac{e A_{0}^{\theta}}{A_{0}}\right. \\
& \left.-b \frac{R_{0}^{\theta}}{B_{0}}\right)\left(3 \frac{A_{0}^{\theta}}{A_{0}}-2 \frac{B_{0}^{\theta}}{B_{0}}+\frac{C_{0}^{\theta}}{C_{0}}\right)+A_{0}^{2}\left[-\frac{2 \epsilon}{A_{0}^{2} B_{0}^{2}}\left(e^{\prime}-\frac{e A_{0}^{\prime}}{A_{0}}-b \frac{R_{0}^{\prime}}{B_{0}}\right)\right]_{, 1} \\
& +A_{0}^{2}\left[\frac{-1}{A_{0}^{2} B_{0}^{2} r^{2}}\left(e^{\theta}-\frac{e A_{0}^{\theta}}{A_{0}}-b \frac{R_{0}^{\theta}}{B_{0}}\right)\right]_{, 2}+\frac{2 \epsilon b}{B_{0}^{3}}\left[R_{0}^{\prime \prime}+\frac{R_{0}^{\theta \theta}}{r^{2}}-2 R_{0}^{\theta}\left(2 \frac{A_{0}^{\prime}}{A_{0}}\right.\right. \\
& \left.\left.+2 \frac{B_{0}^{\prime}}{B_{0}}+\frac{3}{r}\right)-\frac{A_{0}^{\theta} R_{0}^{\theta}}{A_{0} r^{2}}\right]+\frac{2 c \epsilon}{C_{0} B_{0}^{2}}\left[R_{0}^{\prime}\left(\frac{C_{0}^{\prime}}{C_{0}}+\frac{2}{r}-\frac{A_{0}^{\prime}}{A_{0}}\right)+\frac{R_{0}^{\theta}}{r^{2}}\right. \\
& \left.\times\left(\frac{C_{0}^{\theta}}{C_{0}}-\frac{A_{0}^{\theta}}{A_{0}}\right)\right] \\
& P_{1}=-T\left[-\epsilon e R_{0}+2 \frac{\epsilon R_{0}^{\prime}}{B_{0}^{2} r^{2}}\left(e^{\theta}-\frac{2 b R_{0}^{\theta}}{B_{0}}\right)+\frac{2 \epsilon}{B_{0}^{2}}\left(e^{\prime}-\frac{2 b R_{0}^{\prime}}{B_{0}}\right)\left(\frac{A_{0}^{\prime}}{A_{0}}-\frac{B_{0}^{\prime}}{B_{0}}\right.\right. \\
& \left.-\frac{1}{r}+\frac{C_{0}^{\prime}}{C_{0}}\right)+\frac{2 \epsilon}{B_{0}^{2}}\left\{\left(\frac{a}{A_{0}}\right)^{\prime}-\left(\frac{b}{B_{0}}\right)^{\prime}+\left(\frac{c}{C_{0}}\right)^{\prime}\right\}+\frac{2 \epsilon}{B_{0}^{2} r^{2}}\left(e^{\theta}-\frac{2 b R_{0}^{\theta}}{B_{0}}\right) \\
& \left.\times\left(\frac{A_{0}^{\theta}}{A_{0}}-3 \frac{B_{0}^{\theta}}{B_{0}}+\frac{C_{0}^{\theta}}{C_{0}}\right)+\frac{2 \epsilon R_{0}^{\theta}}{B_{0}^{2} r^{2}}\left\{\left(\frac{a}{A_{0}}\right)^{\theta}-\left(\frac{b}{B_{0}}\right)^{\theta}+\left(\frac{c}{C_{0}}\right)^{\theta}\right\}\right] \\
& +\frac{2 \epsilon e \ddot{T}}{A_{0}^{2} B_{0}}+\frac{T}{r^{2} B_{0}^{2}}\left[\{ ( \frac { a } { A _ { 0 } } ) ^ { \theta } + 4 ( \frac { b } { B _ { 0 } } ) ^ { \theta } \} \left(2 \epsilon R_{0}^{\prime \theta}-2 \epsilon \frac{R_{0}^{\theta}}{r}-2 \epsilon R_{0}^{\prime}\right.\right. \\
& \left.\times \frac{B_{0}^{\theta}}{B_{0}}-2 \epsilon R_{0}^{\theta} \frac{B_{0}^{\prime}}{B_{0}}\right)+\left(\frac{A_{0}^{\theta}}{A_{0}}+\frac{4 B_{0}^{\theta}}{B_{0}}\right) 2 \epsilon\left\{-e^{\prime} \frac{B_{0}^{\theta}}{B_{0}}-R_{0}^{\prime}\left(\frac{b}{B_{0}}\right)^{\theta}-\frac{e^{\theta}}{r}\right. \\
& \left.\left.-e^{\theta} \frac{4 B_{0}^{\prime}}{B_{0}}-R_{0}^{\theta}\left(\frac{b}{B_{0}}\right)^{\prime}\right\}\right]-\frac{2 T}{B_{0}^{3} r^{2}}\left[( \frac { A _ { 0 } ^ { \theta } } { A _ { 0 } } + \frac { 4 B _ { 0 } ^ { \theta } } { B _ { 0 } } ) 2 \epsilon \left(R_{0}^{\prime \theta}-\frac{R_{0}^{\theta}}{r}\right.\right. \\
& \left.\left.-R_{0}^{\prime} \frac{B_{0}^{\theta}}{B_{0}}-R_{0}^{\theta} \frac{B_{0}^{\prime}}{B_{0}}\right)\right]+\ddot{T}\left[\frac{-2 \epsilon}{A_{0}^{2}}\left\{e^{\prime}-\frac{A_{0}^{\prime}}{A_{0}} e-\frac{b}{B_{0}} R_{0}^{\prime}\right\}\right]-2 \epsilon T B_{0}^{2} \\
& \times\left[\frac{1}{B_{0}^{2} r^{2}}\left\{e^{\prime} \frac{B_{0}^{\theta}}{B_{0}}+R_{0}^{\prime}\left(\frac{b}{B_{0}}\right)^{\theta}+\frac{e^{\theta}}{r}+e^{\theta} \frac{B_{0}^{\prime}}{B_{0}}+R_{0}^{\theta}\left(\frac{b}{B_{0}}\right)^{\prime}\right\}\right. \\
& \left.+\frac{4 b}{B_{0}^{5} r^{2}}\left\{R_{0}^{\prime 2}-\frac{R_{0}^{\theta}}{r}-R_{0}^{\prime} \frac{B_{0}^{\theta}}{B_{0}}-R_{0} \frac{B_{0}^{\prime}}{B_{0}}\right\}\right]_{, 2}+\frac{2 T \epsilon}{B_{0}}\left(\frac{a}{A_{0}}\right)^{\prime}\left[R_{0}^{\prime \prime}-\frac{R_{0}^{\theta \theta}}{r^{2}}\right. \\
& \left.+\frac{R_{0}^{\theta}}{r^{2}}\left(\frac{B_{0}^{\theta}}{B_{0}}-\frac{A_{0}^{\theta}}{A_{0}}\right)+R_{0}^{\prime}\left(\frac{2}{r}-\frac{B_{0}^{\prime}}{B_{0}}-\frac{A_{0}^{\prime}}{A_{0}}\right)\right]+\frac{2 \epsilon T A_{0}^{\prime}}{A_{0} B_{0}^{2}}\left[e^{\prime \prime}-\frac{2 b R_{0}^{\prime \prime}}{B_{0}}\right.
\end{aligned}
$$




$$
\begin{aligned}
& -\frac{1}{r^{2}}\left(e^{\theta \theta}-\frac{2 b R_{0}^{\theta \theta}}{B_{0}}\right)+\frac{R_{0}^{\theta}}{r^{2}}\left\{\left(\frac{b}{B_{0}}\right)^{\theta}-\left(\frac{a}{A_{0}}\right)^{\theta}\right\}+\frac{1}{r^{2}}\left(e^{\theta}-\frac{2 b R_{0}^{\theta}}{B_{0}}\right) \\
& \times\left(\frac{B_{0}^{\theta}}{B_{0}}-\frac{A_{0}^{\theta}}{A_{0}}\right)+R_{0}^{\prime}\left\{\frac{2}{r}-\left(\frac{b}{B_{0}}\right)^{\prime}-\left(\frac{a}{A_{0}}\right)^{\prime}\right\}+\left(e^{\prime}-\frac{2 b R_{0}^{\prime}}{B_{0}}\right)\left(\frac{2}{r}\right. \\
& \left.\left.-\frac{B_{0}^{\prime}}{B_{0}}-\frac{A_{0}^{\prime}}{A_{0}}\right)\right]+\frac{2 \epsilon}{B_{0}^{2}}\left(\frac{1}{r}+\frac{B_{0}^{\prime}}{B_{0}}\right)\left[e^{\prime \prime}-\frac{2 b R_{0}^{\prime \prime}}{B_{0}}-\frac{1}{r^{2}}\left(e^{\theta \theta}-\frac{2 b R_{0}^{\theta \theta}}{B_{0}}\right)\right. \\
& \left.-\frac{1}{r}\left(e^{\prime}-\frac{2 b R_{0}^{\prime}}{B_{0}}\right)-\frac{2 R_{0}^{\theta}}{r^{2}}\left(\frac{b}{B_{0}}\right)^{\theta}+\frac{2 B_{0}^{\theta}}{B_{0} r^{2}}\left(e^{\theta}-\frac{2 b R_{0}^{\theta}}{B_{0}}\right)\right]+\frac{2 \epsilon T}{B_{0}^{2}} \\
& \times\left(\frac{b}{B_{0}}\right)^{\prime}\left[R_{0}^{\prime \prime}-\frac{R_{0}^{\theta \theta}}{r^{2}}-\frac{R_{0}^{\prime}}{r^{2}}+\frac{R_{0}^{\theta}}{r^{2}}\left(2 B_{0}^{\theta}\right)\right]-\frac{2 \epsilon T C_{0}^{\prime}}{B_{0}^{2} C_{0}}\left[e^{\prime \prime}-\frac{2 b R_{0}^{\prime \prime}}{B_{0}}\right. \\
& +\frac{R_{0}^{\theta}}{r^{2}}\left\{\left(\frac{b}{B_{0}}\right)^{\theta}-\left(\frac{c}{C_{0}}\right)^{\theta}\right\}+\left(e^{\theta}-\frac{2 b R_{0}^{\theta}}{B_{0}}\right)\left(\frac{B_{0}^{\theta}}{B_{0}}-\frac{C_{0}^{\theta}}{C_{0}}\right)-R_{0}^{\prime} \\
& \left.\times\left\{\left(\frac{b}{B_{0}}\right)^{\prime}+\left(\frac{c}{C_{0}}\right)^{\prime}\right\}-\left(e^{\prime}-\frac{2 b R_{0}^{\prime}}{B_{0}}\right)\left(\frac{B_{0}^{\prime}}{B_{0}}+\frac{C_{0}^{\prime}}{C_{0}}\right)\right]-\frac{2 \epsilon T}{B_{0}^{2}}\left(\frac{c}{C_{0}}\right)^{\prime} \\
& \times\left[R_{0}^{\prime \prime}+\frac{R_{0}^{\theta}}{r^{2}}\left(\frac{B_{0}^{\theta}}{B_{0}}-\frac{C_{0}^{\theta}}{C_{0}}\right)-R_{0}^{\prime}\left(\frac{B_{0}^{\prime}}{B_{0}}+\frac{C_{0}^{\prime}}{C_{0}}\right)\right], \\
& D_{5}=T B_{0}^{2}\left[\frac{2 \epsilon}{B_{0}^{4} r^{4}}\left\{e^{\prime \theta}-\frac{e^{\theta}}{r}-R_{0}^{\prime}\left(\frac{b}{B_{0}}\right)^{\theta}-e^{\prime} \frac{B_{0}^{\theta}}{B_{0}}-R_{0}^{\theta}\left(\frac{b}{B_{0}}\right)^{\prime}\right\}\right. \\
& \left.-\frac{8 \epsilon b}{B_{0}^{5} r^{4}}\left(R_{0}^{\prime \theta}-\frac{R_{0}^{\theta}}{r}-R_{0}^{\prime} \frac{B_{0}^{\theta}}{B_{0}}-R_{0}^{\theta} \frac{B_{0}^{\prime}}{B_{0}}\right)\right]_{, 1}+4 T b \epsilon B_{0}\left[\frac{1}{B_{0}^{4} r^{2}}\right. \\
& \left.\times\left(R_{0}^{\prime \theta}-\frac{R_{0}^{\theta}}{r}-R_{0}^{\prime} \frac{B_{0}^{\theta}}{B_{0}}-R_{0}^{\theta} \frac{B_{0}^{\prime}}{B_{0}}\right)\right]_{, 1}+\frac{2 \epsilon T B_{0}^{\theta}}{B_{0}^{3} r^{2}}\left[\frac{1}{r^{2}}\left(e^{\theta \theta} \frac{2 b R_{0}^{\theta \theta}}{B_{0}}\right)\right. \\
& -e^{\prime \prime}+\frac{2 b R_{0}^{\prime \prime}}{B_{0}}-\frac{1}{r}\left(e^{\prime}-\frac{2 b R_{0}^{\prime}}{B_{0}}\right)-\frac{2 R_{0}^{\theta}}{r^{2}}\left(\frac{b}{B_{0}}\right)^{\theta}-\frac{2}{r^{2}}\left(e^{\theta}-\frac{2 b R_{0}^{\theta}}{B_{0}}\right) \\
& \left.\times \frac{B_{0}^{\theta}}{B_{0}}\right]+\frac{2 \epsilon T}{B_{0}^{2} r^{2}}\left(\frac{b}{B_{0}}\right)^{\theta}\left[\frac{R_{0}^{\theta \theta}}{r^{2}}-R_{0}^{\prime \prime}-\frac{R_{0}^{\prime}}{r}-\frac{2 R_{0}^{\theta}}{r^{2}}\left(\frac{B_{0}^{\theta}}{B_{0}}\right)\right]-\frac{2 \epsilon T}{B_{0}^{2} r^{2}} \\
& \times\left(\frac{a}{A_{0}}\right)^{\theta}\left[\frac{R_{0}^{\theta \theta}}{r^{2}}+R_{0}^{\prime}\left(\frac{1}{r}-\frac{B_{0}^{\prime}}{B_{0}}-\frac{A_{0}^{\prime}}{A_{0}}\right)-\frac{R_{0}^{\theta}}{r^{2}}\left(\frac{A_{0}^{\theta}}{A_{0}}+\frac{B_{0}^{\theta}}{B_{0}}\right)\right] \\
& +\frac{2 \epsilon T A_{0}^{\theta}}{A_{0} B_{0}^{2} r^{2}}\left[\frac{1}{r^{2}}\left(e^{\theta \theta}-\frac{2 b R_{0}^{\theta \theta}}{B_{0}}\right)-R_{0}^{\prime}\left\{\left(\frac{b}{B_{0}}\right)^{\prime}+\left(\frac{a}{A_{0}}\right)^{\prime}\right\}\right. \\
& +\left(e^{\prime}-\frac{2 b R_{0}^{\prime}}{B_{0}}\right)\left(\frac{1}{r}-\frac{B_{0}^{\prime}}{B_{0}}-\frac{A_{0}^{\prime}}{A_{0}}\right)-\frac{R_{0}^{\theta}}{r^{2}}\left\{\left(\frac{b}{B_{0}}\right)^{\theta}+\left(\frac{a}{A_{0}}\right)^{\theta}\right\} \\
& \left.-\frac{1}{r^{2}}\left(e^{\theta}-\frac{2 b R \theta_{0}}{B_{0}}\right)\left(\frac{A_{0}^{\theta}}{A_{0}}+\frac{B_{0}^{\theta}}{B_{0}}\right)\right]+\frac{2 \epsilon e A_{0}^{\prime} \ddot{T}}{A_{0}^{3} r^{2}}+\frac{2 \epsilon}{B_{0}^{2} r^{2}}\left(R_{0}^{\prime \theta}\right.
\end{aligned}
$$




$$
\begin{aligned}
& \left.-\frac{R_{0}^{\theta}}{r}-R_{0}^{\prime} \frac{B_{0}^{\theta}}{B_{0}}-R_{0}^{\theta} \frac{B_{0}^{\prime}}{B_{0}}\right)\left\{\left(4 \frac{b}{B_{0}}\right)^{\prime}+\left(\frac{a}{A_{0}}\right)^{\prime}+\left(\frac{c}{C_{0}}\right)^{\prime}\right\} \\
& +\frac{2 T \epsilon}{B_{0}^{2} r^{2}}\left\{e^{\prime \theta}-\frac{e^{\theta}}{r}-R_{0}^{\prime}\left(\frac{b}{B_{0}}\right)^{\theta}-e^{\prime} \frac{B_{0}^{\theta}}{B_{0}}-R_{0}^{\theta}\left(\frac{b}{B_{0}}\right)^{\prime}\right\}\left(\frac{A_{0}^{\prime}}{A_{0}}\right. \\
& \left.+\frac{3}{r}+4 \frac{B_{0}^{\prime}}{B_{0}}+\frac{C_{0}^{\prime}}{C_{0}}\right)-\frac{4 \epsilon T b}{B_{0}^{3} r^{2}}\left(R_{0}^{\prime \theta}-\frac{R_{0}^{\theta}}{r}-R_{0}^{\prime} \frac{B_{0}^{\theta}}{B_{0}}-R_{0}^{\theta} \frac{B_{0}^{\prime}}{B_{0}}\right) \\
& \times\left(\frac{A_{0}^{\prime}}{A_{0}}+\frac{3}{r}+4 \frac{B_{0}^{\prime}}{B_{0}}+\frac{C_{0}^{\prime}}{C_{0}}\right)-\frac{2 \epsilon T}{r^{2}}\left[\frac { 1 } { B _ { 0 } ^ { 2 } } \left\{-\frac{e R_{0} B_{0}^{2}}{2}+e^{\prime \prime}\right.\right. \\
& -\frac{2 b R_{0}^{\prime \prime}}{B_{0}}+R_{0}^{\prime}\left\{\left(\frac{a}{A_{0}}\right)^{\prime}-\left(\frac{b}{B_{0}}\right)^{\prime}+\left(\frac{c}{C_{0}}\right)^{\prime}\right\}+\left(e^{\prime}-\frac{2 b R_{0}^{\prime}}{B_{0}}\right) \\
& \times\left(\frac{A_{0}^{\prime}}{A_{0}}-\frac{B_{0}^{\prime}}{B_{0}}+\frac{C_{0}^{\prime}}{C_{0}}\right)+\frac{R_{0}^{\theta}}{r^{2}}\left\{\left(\frac{a}{A_{0}}\right)^{\theta}-\left(\frac{b}{B_{0}}\right)^{\theta}+\left(\frac{c}{C_{0}}\right)^{\theta}\right\} \\
& \left.\left.+\frac{1}{r^{2}}\left(\frac{A_{0}^{\theta}}{A_{0}}-\frac{B_{0}^{\theta}}{B_{0}}+\frac{C_{0}^{\theta}}{C_{0}}\right)\right\}\right]_{, 2}+\frac{2 \epsilon C_{0}^{\theta}}{C_{0} B_{0}^{2} r^{2}}\left[\frac{1}{r^{2}}\left(e^{\theta \theta}-\frac{2 b R_{0}^{\theta \theta}}{B_{0}}\right)\right. \\
& -R_{0}^{\prime}\left\{\left(\frac{b}{B_{0}}\right)^{\prime}+\left(\frac{c}{C_{0}}\right)^{\prime}\right\}+\left(e^{\prime}-\frac{2 b R_{0}^{\prime}}{B_{0}}\right)\left(\frac{B_{0}^{\prime}}{B_{0}}+\frac{1}{r}+\frac{C_{0}^{\prime}}{C_{0}}\right) \\
& \left.-\frac{R_{0}^{\theta}}{r^{2}}\left\{\left(\frac{b}{B_{0}}\right)^{\theta}+\left(\frac{c}{C_{0}}\right)^{\theta}\right\}+\frac{1}{r^{2}}\left(e^{\theta}-\frac{2 b R_{0}^{\theta}}{B_{0}}\right)\left(\frac{B_{0}^{\theta}}{B_{0}}+\frac{C_{0}^{\theta}}{C_{0}}\right)\right] \\
& +\frac{2 \epsilon T}{B_{0}^{2} r^{2}}\left[\frac{R_{0}^{\theta \theta}}{r^{2}}-R_{0}^{\prime}\left(\frac{B_{0}^{\prime}}{B_{0}}+\frac{1}{r}+\frac{C_{0}^{\prime}}{C_{0}}\right)-\frac{R_{0}^{\theta}}{r^{2}}\left(B_{0}^{\theta}+\frac{C_{0}^{\theta}}{C_{0}}\right)\right] .
\end{aligned}
$$

The coefficient $\delta^{2}$ of the differential equation (41) is given by

$$
\begin{aligned}
\delta^{2} & =\frac{-A_{0}^{2}}{2}\left[e-\frac{2}{B_{0}^{2}}\left\{\frac{C_{0}^{\prime} A_{0}^{\prime}}{C_{0} A_{0}}\left(\frac{a^{\prime}}{A_{0}^{\prime}}-\frac{a}{A_{0}}+\frac{c^{\prime}}{C_{0}^{\prime}}-\frac{c}{C_{0}}\right)+\left(\frac{a^{\prime \prime}}{A_{0}^{\prime \prime}}-\frac{a}{A_{0}}\right) \frac{A_{0}^{\prime \prime}}{A_{0}}\right.\right. \\
& +\left(\frac{b^{\prime \prime}}{B_{0}^{\prime \prime}}-\frac{b}{B_{0}}\right) \frac{B_{0}^{\prime \prime}}{B_{0}}-\frac{1}{r}\left(\frac{a}{A_{0}}-\frac{c}{C_{0}}-\frac{b}{B_{0}}\right)^{\prime}+\left(\frac{\bar{c}^{\prime \prime}}{C_{0}^{\prime \prime}}-\frac{\bar{c}}{C_{0}}\right) \frac{C_{0}^{\prime \prime}}{C_{0}}-2\left(\frac{b}{B_{0}}\right)^{\prime} \\
& \times \frac{B_{0}^{\prime}}{B_{0}}+\left(\frac{b}{B_{0}}\right)^{\theta} \frac{B_{0}^{\theta}}{B_{0}} \frac{2}{r^{2}}+\left(\frac{c^{\theta \theta}}{C_{0}^{\theta \theta}}-\frac{c}{C_{0}}\right) \frac{C_{0}^{\theta \theta}}{C_{0}}+\left(\frac{b^{\theta \theta}}{B_{0}^{\theta \theta}}-\frac{b}{B_{0}}\right) \frac{B_{0}^{\theta \theta}}{B_{0}}+\left(\frac{a^{\theta \theta}}{A_{0}^{\theta \theta}}\right. \\
& \left.\left.\left.-\frac{a}{A_{0}}\right) \frac{A_{0}^{\theta \theta}}{A_{0}}+\frac{C_{0}^{\theta} A_{0}^{\theta}}{C_{0} A_{0}}\left(\frac{a^{\theta}}{A_{0}^{\theta}}-\frac{a}{A_{0}}+\frac{c^{\theta}}{C_{0}^{\theta}}-\frac{c}{C_{0}}\right)\right\}+2 \frac{R_{0} b}{B_{0}}\right]\left(\frac{b}{B_{0}}-\frac{\bar{c}}{C_{0}}\right)^{-1} .
\end{aligned}
$$




\section{REFERENCES}

Allen, S.W., Schmidt, R.W., Ebeling, H., Fabian, A.C. and Speybroeck, L.V.: Mon. Not. R. Astron. Soc. 353, 457 (2004)

Amendola, L., Gannouji, R., Polarski, D. and Tsujikawa, S.: Phys. Rev. D 75, 083504 (2007)

Bennett, C.L. et al.: Astrophys. J. Suppl. Ser. 148, 1 (2003)

Bergliaffa, S.E.P. and Nunes, Y.E.C.O.: Phys. Rev. D 84, 084006 (2011)

Capozziello, S.: Int. J. Mod. Phys. D 11, 483 (2002)

Capozziello, S. and Laurentis, M.D.: Phys. Rep. 509, 167 (2011)

Capozziello, S., De Laurentis, M., Odintsov, S.D. and Stabile, A.: Phys. Rev. D 83, 064004 (2011)

Capozziello, S., De Laurentis, M., De Martino, I., Formisano, M. and Odintsov, S.D.: Phys. Rev. D 85, 044022 (2012)

Cembranos, J.A.R., Dombriz, A.D.L.C. and Núñez, B.M.: J. Cosmol. Astropart. Phys. 04, $021(2012)$

Chakraborty, S., Chakraborty, S. and Debnath, U.: Int. J. Mod. Phys. D 14, 1707 (2005)

Chandrasekhar, S.: Astrophys. J. 140, 417 (1964)

Chan, R.: Mon. Not. R. Astron. Soc. 316(2000)588

Chan, R., Herrera, L. and Santos, N.O.: Mon. Not. R. Astron. Soc. 265, 533 (1993)

Chan, R., Herrera, L. and Santos, N.O.: Mon. Not. R. Astron. Soc. 267, 637 (1994)

Cognola, G., Elizalde, E., Nojiri, S., Odintsov, S.D. and Zerbini, S.: J. Cosmol. Astropart. Phys. 02, 010 (2005)

Cognola, G., Elizalde, E., Nojiri, S., Odintsov, S.D., Sebastiani, L. and Zerbini, S.: Phys. Rev. D 77, 046009 (2008)

Copeland, E.J., Sami, M. and Tsujikawa, S.: Int. J. Mod. Phys. D 15, 1753 (2006)

Cruz-Dombriz, A.D.L. and Sáaez-Gómez, D.: Entropy 14, 1717 (2012)

Cruz-Dombriz, A.D.L., Dobado, A. and Maroto, A.L.: Phys. Rev. D 80, 124011 (2009) 
Farinelli, R, De Laurentis, M., Capozziello, S. and Odintsov, S.D.: arXiv:1311.2744 (2014)

Felice, A.D. and Tsujikawa, S.: Living Rev. Relativity 13, 3 (2010)

Garattini R.: J. Phys.: Conf. Ser., 174, 012066 (2009)

Harrison, B.K., Thorne, K.S., Wakano, M. and Wheeler, J.A.: Gravitation Theory and Gravitational Collapse (University of Chicago Press, 1965)

Herrera, L. and Santos, N.O.: Phys. Rep. 286, 53 (1997)

Herrera, L. and Santos, N.O.: Astrophys. J. 438, 308 (1995)

Herrera, L., Santos, N.O. and Le Denmat, G.: Mon. Not. R. Astron. Soc. 237, 257 (1989)

Herrera, L., Di Prisco, A.: J. Ospino and J. Ibáñez, Phys. Rev. D 87, 024014 (2013)

Herrera, L., Di Prisco, A., Iba nez, J., Ospino, J.: Phys. Rev. D 89, 084034 (2014)

Huang, Q.G.: J. Cosmol. Astropart. Phys., 02, 035 (2014)

Jain, B. and Taylor, A.: Phys. Rev. Lett. 91, 141302 (2003)

Mak, M.K. and Harko, T.: R. Soc. London A 459, 393 (2003)

Matarrese, S. and Terranova, D.: Mon. Not. R. Astron. Soc. 283, 400 (1996)

Misner, C.W. and Sharp, D.: Phys. Rev. 136, B571 (1964)

Nojiri, S. and Odintsov, S.D.: Int. J. Geom. Meth. Mod. Phys. 4, 115 (2007)

Nojiri, S. and Odintsov, S.D.: Phys. Rep. 505, 59 (2011)

Nojiri, S. and Odintsov, S.D.: Class. Quantum Grav. 30, 125003 (2013)

Oppenheimer, J.R. and Snyder, H.: Phys. Rev. 56, 455 (1939)

Perlmutter, S. et al.: Nature 391, 51 (1998)

Riess, A.G. et al.: Astrophys. J. 116, 1009 (1998)

Roy, S.R. and Tripathi, V.N.: Gen. Realtiv. Gravit. 2, 121 (1971)

Sebastiani, L., Momeni, D., Myrzakulov, R. and Odintsov, S.D.: Phys. Rev. D 88, 104022 (2013)

Sharif, M. and Bhatti, M.Z.: Gen. Relativ. Gravit. 44, 2811 (2012a). 
Sharif, M. and Bhatti, M.Z.: Mod. Phys. Lett. A 27, 1250141 (2012b)

Sharif, M. and Bhatti, M.Z.: J. Cosmol. Astropart. Phys. 10, 056 (2013a)

Sharif, M. and Bhatti, M.Z.: Phys. Lett. A 378, 469 (2014)

Sharif, M. and Bhatti, M.Z.: J. Cosmol. Astropart. Phys. 11, 014 (2013b)

Sharif, M. and Yousaf, Z.: Int. J. Mod. Phys. D 21, 1250095 (2012a)

Sharif, M. and Yousaf, Z.: Can. J. Phys. 90, 865 (2012b)

Sharif, M. and Yousaf, Z.: Mon. Not. R. Astron. Soc. 432, 264 (2013)

Sharif, M. and Yousaf, Z.: Phys. Rev. D 88(2013a)024020.

Sharif, M. and Yousaf, Z.: Astrophys. Space Sci. 351, 351 (2014a)

Sharif, M. and Yousaf, Z.: Astrophys. Space Sci. DOI 10.1007/s10509-014-1913-z (2014b)

Sotirou, T.P. and Faraoni, V.: Rev. Mod. Phys. 82, 451 (2010)

Starobinsky, A.A.: Phys. Lett. B 91, 99 (1980)

Weber, F.: Pulsars as Astrophysical Observatories for Nuclear and Particle Physics, IOP Publishing, Bristol, (1999)

Ziaie A. H., Atazadeh K. and Rasouli S.M.M.: Gen. Relativ. Gravit., 43, 2943 (2011) 